\title{
EXISTENCE AND LIMITING BEHAVIOR OF A NON-INTERIOR- POINT TRAJECTORY FOR NONLINEAR COMPLEMENTARITY PROBLEMS WITHOUT STRICT FEASIBILITY CONDITION*
}

\author{
YUN-BIN ZHAO ${ }^{\dagger}$ AND DUAN LI
}

\begin{abstract}
For $\mathrm{P}_{0}$-complementarity problems, most existing non-interior-point path-following methods require the existence of a strictly feasible point. (For a $\mathrm{P}_{*}$-complementarity problem, the existence of a strictly feasible point is equivalent to the nonemptyness and the boundedness of the solution set). In this paper, we propose a new homotopy formulation for complementarity problems by which a new non-interior-point continuation trajectory is generated. The existence and the boundedness of this non-interior-point trajectory for $\mathrm{P}_{0}$-complementarity problems are proved under a very mild condition that is weaker than most used conditions in the literature. One prominent feature of this condition is that it may hold even when the often-assumed strict feasibility condition

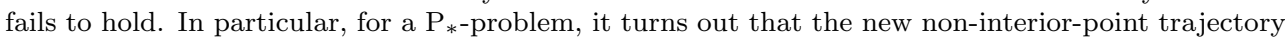
exists and is bounded if and only if the problem has a solution. We also study the convergence of this trajectory and the characterization of its limiting point as the parameter approaches zero.
\end{abstract}

Key words. Complementarity problems, non-interior-point methods, homotopy continuation trajectories, $\mathrm{P}_{0}$-functions, $\mathrm{P}_{*}$-functions

AMS subject classifications. 90C30, 90C33, 65K10

1. Introduction. The standard complementarity problem (abbreviated, $\mathrm{CP}$ ) is to find a pair $(x, y) \in R^{n} \times R^{n}$ such that

$$
y=f(x),(x, y) \geq 0 \text { and } x^{T} y=0,
$$

where $f: R^{n} \rightarrow R^{n}$ is a continuous function. This problem has many applications in optimization, economics, and engineering. See for example, Cottle, Pang, and Stone [8], Harker and Pang [14], Heemels, Schumacher, and Weiland [15], van der Schaft and Schumacher [37], and Lötstedt [23].

The first non-interior-point method for the $\mathrm{CP}$ was proposed by Chen and Harker [5], and was based on the use of Chen-Harker-Kanzow-Smale smooth function. Due to the impressive numerical performance of the algorithm as well as its ideal convenience for the application to those $\mathrm{CPs}$ where interiority restriction on the iterates is quite severe, there are a growing interest and fruitful results in the non-interior-point methods for the CP, see, e.g., Kanzow [18], Burke and Xu [1, 2, 3, 4], Xu [35], Xu and Burke [36], Chen and Chen [6], Hotta and Yoshise [16], Hotta, Inaba, and Yoshise [17], Qi and Sun [26], and Tseng [32]. In the setting of $\mathrm{P}_{0^{-}} \mathrm{CPs}$, a common feature of the above-mentioned non-interior-point methods is to make an assumption of the strict feasibility condition (or the nonemptyness and the boundedness conditions on the solution set) and a proper condition. For instance, Hotta and Yoshise [16] utilized the following condition.

${ }^{*}$ The work was partially supported by grant CUHK4392/99E, research Grants Council, Hong Kong.

$\dagger$ Department of Systems Engineering and Engineering Management, The Chinese University of Hong Kong, Shatin, NT, Hong Kong, and Institute of Applied Mathematics, Chinese Academy of Sciences, Beijing, China (Email: ybzhao@se.cuhk.edu.hk).

${ }^{\ddagger}$ Corresponding author. Department of Systems Engineering and Engineering Management, The Chinese University of Hong Kong, Shatin, NT, Hong Kong (Fax: (852) 26035505; Tel: (852) 26098323; Email: dli@se.cuhk.edu.hk). 
Condition 1.1. (i) $f$ is a $P_{0}$-function, i.e., for any distinct vectors $x, y$ in $R^{n}$

$$
\max _{x_{i} \neq y_{i}}\left(x_{i}-y_{i}\right)\left(f_{i}(x)-f_{i}(y)\right) \geq 0 \text {. }
$$

(ii) There exists a strictly feasible point $\left(x^{0}, y^{0}\right)$, i.e., $x^{0}>0$ and $y^{0}=f\left(x^{0}\right)>0$.

(iii) The set

$$
U^{-1}(D)=\left\{(u, x, y) \in R_{+}^{n} \times R^{2 n}: U(u, x, y) \in D\right\}
$$

is bounded for every compact subset $D$ of $R_{+}^{n} \times V\left(R_{++}^{n} \times R^{2 n}\right)$, where $V: R_{+}^{n} \times R^{2 n} \rightarrow$ $R^{n}$ and $U: R_{+}^{n} \times R^{2 n} \rightarrow R_{+}^{n} \times R^{2 n}$ are given by

$$
V(u, x, y)=x+y-\sqrt{(x-y)^{2}+4 u}
$$

and

$$
U(u, x, y)=\left(\begin{array}{c}
u \\
x+y-\sqrt{(x-y)^{2}+4 u} \\
y-f(x)
\end{array}\right)=\left(\begin{array}{c}
u \\
V(u, x, y) \\
y-f(x)
\end{array}\right)
$$

respectively. All the above algebraic operations are performed componentwise.

The following standard condition was widely used in interior-point methods and non-interior-point methods. See for example, [2, 3, 6, 16, 17, 19, 20, 21, 26, 38].

CONDITION 1.2. (i) $f$ is monotone, i.e., $(x-y)^{T}(f(x)-f(y)) \geq 0$ for any $(x, y) \in R^{2 n}$.

(ii) There exists a strictly feasible point $\left(x^{0}, y^{0}\right)$, i.e., $x^{0}>0$ and $y^{0}=f\left(x^{0}\right)>0$.

Condition 1.2 implies Condition 1.1 (see, $[16,26]$ ). Hotta and Yoshise [16] pointed out that Condition 1.1 implies the well known Condition 1.5 in Kojima, Megiddo, and Noma [19]. As observed by Zhao and Li [42] (see also section 3 of this paper), the Condition 1.5 in [19] implies that the solution set of the CP is nonempty and bounded. Thus, the above-mentioned Conditions 1.1 and 1.2 all imply that the solution set of the CP is nonempty and bounded. Ravindran and Gowda (Corollary 5 in [27]) showed that a $\mathrm{P}_{0}-\mathrm{CP}$ with a nonempty and bounded solution set must have a strictly feasible point. Moreover, for monotone CPs, the converse is also true, i.e., the solution set of the monotone $\mathrm{CP}$ is nonempty and bounded if and only if it has a strictly feasible point. (See also Chen, Chen, and Kanzow [7]). This property of the monotone problem can be extended to the case of $\mathrm{P}_{*}$-CPs. We recall that a map $f: R^{n} \rightarrow R^{n}$ is said to be a $\mathrm{P}_{*}$-function if there exists a constant $\tau \geq 0$ such that

$$
(1+\tau) \sum_{i \in I_{+}}\left(x_{i}-y_{i}\right)\left(f_{i}(x)-f_{i}(y)\right)+\sum_{i \in I_{-}}\left(x_{i}-y_{i}\right)\left(f_{i}(x)-f_{i}(y)\right) \geq 0
$$

for all distinct vectors $x, y$ in $R^{n}$, where $I_{+}=\left\{i:\left(x_{i}-y_{i}\right)\left(f_{i}(x)-f_{i}(y)\right)>0\right\}$ and $I_{-}=\{1, \ldots, n\} \backslash I_{+}$. (See, Cottle, Pang, and Venkateswaran [9], Kojima et al. [20], Väliaho [33], Zhao and Han [38], and Zhao and Isac [39, 40]). Clearly, a monotone function is a $\mathrm{P}_{*}$-function, but the converse is not true. Zhao and $\mathrm{Li}[41,42]$ pointed out that for a $\mathrm{P}_{*}-\mathrm{CP}$ the following three conditions are equivalent:

(i) There exists a strictly feasible point.

(ii) The solution set of the CP is nonempty and bounded.

(iii) The central path of the $\mathrm{CP}$ exists. 
Since most existing (interior-point and) non-interior-point path-following algorithms for CPs are based on the use of certain continuation trajectory such as the central path whose existence is closely related to the existence of a strictly feasible point, we conclude that for $\mathrm{P}_{0}$-CPs these (interior-point and) non-interior-point algorithms are, in fact, confined to solving a class of strictly feasible problems. Other non-interior-point algorithms in the literature also suffer from the same restriction. For instance, the algorithms developed by Chen and Harker [5], Burke and Xu [1], and Chen and Chen [6] require the $\mathrm{P}_{0}$ and $\mathrm{R}_{0}$ assumption, which also implies that the solution set of the CP is nonempty and bounded, and hence the problem is strictly feasible. The strict feasibility condition plays an indispensable role in these known non-interior-point methods. In section 3, we gave an example to show that the Hotta and Yoshise's non-interior-point trajectory [16] does not necessarily exist when the problem has no strictly feasible point, in which case the solution set of the $\mathrm{P}_{0}$-CP is unbounded provided that it is nonempty). An interesting question is how to circumvent this difficulty so that a non-interior-point path-following method can be designed to solve a CP even when there is no strictly feasible point.

In this paper, we shall propose a new homotopy formulation of the CP. Based on this formulation, a new non-interior-point continuation trajectory for the CP can be generated. This new continuation trajectory possesses a desirable feature: For $\mathrm{P}_{0}$-CPs, the existence and the boundedness of the continuation trajectory can be ensured under a mild condition that is weaker than most existing conditions such as Conditions 1.1 and 1.2. The often-assumed strict feasibility condition is not required here. Particularly, for $\mathrm{P}_{*}$-CPs, the proposed continuation trajectory exists and is bounded if and only if the problem has a solution. In other words, the existence and the boundedness of the trajectory for $\mathrm{P}_{*}$-CPs do not require the strict feasibility condition which is equivalent to the nonempty and the boundedness of the solution set. We also (i) provide some sufficient conditions for the convergence of the entire trajectory as the parameter approaches zero, and (ii) identify the properties of the limiting point of this trajectory. The results presented in the paper bring us with a theoretical basis for devising a new non-interior-point path-following method for CPs. This method is expected to solve a general class of complementarity problems which is broader than those to which most existing methods can be applied.

This paper is organized as follows: In section 2, we define a new homotopy formulation for the CP. In section 3 , we specify a new proper condition that will be used to prove the existence and boundedness of a new continuation trajectory in section 4. We also compare this condition with several known ones in the literature. The limiting behavior of the trajectory is studied in section 5 . Final remarks are given in section 6 .

Notation: We denote by $R^{n}$ the space of $n$-dimensional real vectors, and by $R_{+}^{n}\left(R_{++}^{n}\right.$, respectively) the nonnegative orthant (positive orthant, respectively). If $x \in R_{+}^{n}\left(R_{++}^{n}\right)$, we write $x \geq 0(x>0)$ for simplicity. All vectors, unless otherwise stated, are column vectors. $T$ denotes the transpose of a vector. The symbol $e$ denotes the vector of all ones in $R^{n}$. For given vectors $u, w, v$ in $R^{n}$, the triplet $(u, w, v)$ (the pair $(x, y))$ denotes the column vector $\left(u^{T}, w^{T}, v^{T}\right)^{T}\left(\left(x^{T}, y^{T}\right)^{T}\right)$. For any $u \in R_{+}^{n}$, the symbol $u^{p}$ denotes the $p$ th power of the vector $u$, i.e., the vector $\left(u_{1}^{p}, \ldots, u_{n}^{p}\right)^{T}$ where $p>0$ is a positive scalar. In particular, when $p=1 / 2, \sqrt{u}$ denotes the vector $\left(\sqrt{u_{1}}, \ldots, \sqrt{u_{n}}\right)^{T}$. The symbol $\operatorname{diag}(x)$ denotes the $n \times n$ diagonal matrix whose $(i, i)$ th entry is $x_{i}$. For any $x, y \in R^{n}$ with $x \leq y$, we denote the rectangular box $\left[x_{1}, y_{1}\right] \times \cdots \times\left[x_{n}, y_{n}\right]$ by $[x, y]$. 
2. A new homotopy formulation for CPs. Let $(\bar{u}, \bar{v}, \bar{r})$ be a fixed point in $R_{++}^{n} \times R^{2 n}$ and let

$$
\bar{w}=\left\{\theta(\bar{u}, \bar{v}, \bar{r}) \in R_{++}^{n} \times R^{2 n}: \theta \in(0,1]\right\} .
$$

Let $U: R_{+}^{n} \times R^{2 n} \rightarrow R^{3 n}$ be defined by (1.1). Denote

$$
U^{-1}(\bar{w})=\left\{z=(u, x, y) \in R_{++}^{n} \times R^{2 n}: U(z)=\theta(\bar{u}, \bar{v}, \bar{r}) \text { for some } \theta \in(0,1]\right\} .
$$

Under Condition 1.1, Hotta and Yoshise [16] showed that the above set forms a continuous trajectory leading to a solution of the CP. Based on this fact, they designed a globally convergent path-following method for the CP. However, it is easy to see that the strict feasibility condition plays an essential role in the existence of the Hotta and Yoshise trajectory. In fact, it is impossible to remove the strict feasibility condition from Condition 1.1 and Condition 1.2 without destroying the existence of their trajectory, as we see in the following example.

EXAMPLE 2.1. Let $f(x)=M x+q$ where

$$
M=\left(\begin{array}{cc}
0 & -1 \\
0 & 0
\end{array}\right), \quad q=\left(\begin{array}{c}
-1 \\
0
\end{array}\right) .
$$

This function is a $\mathrm{P}_{0}$-function and there exists no strictly feasible point. The solution set of the corresponding CP is unbounded. Let $\bar{u}=\left(\bar{u}_{1}, \bar{u}_{2}\right)^{T} \in R_{++}^{2}, \bar{v}=\left(\bar{v}_{1}, \bar{v}_{2}\right)^{T} \in$ $R^{2}$ and $\bar{r}=\left(\bar{r}_{1}, \bar{r}_{2}\right)^{T} \in R^{2}$. From Lemma 1.1 in [16], the system $U(u, x, y)=\theta(\bar{u}, \bar{v}, \bar{r})$ can be written as follows:

$$
\begin{aligned}
& u=\theta \bar{u}, y=f(x)+\theta \bar{r}, x-\theta \bar{v} / 2>0, y-\theta \bar{v} / 2>0, \\
& \operatorname{diag}(x-\theta \bar{v} / 2)(y-\theta \bar{v} / 2)=\theta \bar{u} .
\end{aligned}
$$

Note that $y=f(x)+\theta \bar{r}=\left(\begin{array}{c}-x_{2}-1+\theta \bar{r}_{1} \\ \theta \bar{r}_{2}\end{array}\right)$. The last equation above can be rewritten as

$$
\begin{gathered}
\left(x_{1}-\theta \bar{v}_{1} / 2\right)\left(-x_{2}-1+\theta \bar{r}_{1}-\theta \bar{v}_{1} / 2\right)=\theta \bar{u}_{1}, \\
\left(x_{2}-\theta \bar{v}_{2} / 2\right)\left(\theta \bar{r}_{2}-\theta \bar{v}_{2} / 2\right)=\theta \bar{u}_{2} .
\end{gathered}
$$

Since $\theta>0$, the second equation above reduces to

$$
\left(x_{2}-\theta \bar{v}_{2} / 2\right)\left(\bar{r}_{2}-\bar{v}_{2} / 2\right)=\bar{u}_{2} .
$$

Case 1: $\bar{r}_{2} \leq \bar{v}_{2} / 2$. Since $x_{2}-\theta \bar{v}_{2} / 2>0$ and $\bar{u}_{2}>0$, the above equation has no solution, and thus the system $U(u, x, y)=\theta(\bar{u}, \bar{v}, \bar{r})$ has no solution.

Case 2: $\bar{r}_{2}>\bar{v}_{2} / 2$. In this case, $(2.2)$ can be written as

$$
x_{2}=\left(\bar{r}_{2}-\bar{v}_{2} / 2\right)^{-1} \bar{u}_{2}+\theta \bar{v}_{2} / 2 .
$$

Hence, for all sufficiently small $\theta>0$, we have

$$
-x_{2}-1+\theta \bar{r}_{1}-\theta \bar{v}_{1} / 2=-\left(\bar{r}_{2}-\bar{v}_{2} / 2\right)^{-1} \bar{u}_{2}-\theta \bar{v}_{2} / 2-1+\theta \bar{r}_{1}-\theta \bar{v}_{1} / 2<0 .
$$

Since $x_{1}-\theta \bar{v}_{1} / 2>0$, we deduce from the above that the equation (2.1) has no solution for all sufficiently small $\theta>0$. Thus, the Hotta-Yoshise trajectory [16] does not exist. 
Motivated by the above example, we now introduce a new homotopy formulation for the CP. Let $p \in(0, \infty)$ and $q \in[1, \infty)$ be two fixed numbers throughout the paper. Define the homotopy map $H: R_{+}^{n} \times R^{2 n} \rightarrow R^{3 n}$ as follows:

$$
H(u, x, y)=\left(\begin{array}{c}
u \\
x+y-\sqrt{(x-y)^{2}+4 u^{q}} \\
y-\left(f(x)+\operatorname{diag}\left(u^{p}\right) x\right)
\end{array}\right),(u, x, y) \in R_{+}^{n} \times R^{2 n}
$$

The above homotopy map is the focus of our study. It is worth mentioning that for each fixed vector $u>0$, the function $f(x)+\operatorname{diag}\left(u^{p}\right) x$ can be viewed as a form of the renowned Tikhonov regularization of $f$, which was originally utilized to handle ill-posed problems. Recently, more attentions have been paid to such a technique, see, e.g., Venkateswaran [34], Facchinei [10], Facchinei and Kanzow [11], Facchinei and Pang [12], Ravindran and Gowda [27], Gowda and Tawhid [13], Sznajder and Gowda [29], Qi [25], Sun [28], Tseng [31], and Zhao and Li [42]. To deal with the case of nonexistence of a strictly feasible point (or unboundedness of the solution set), we will see from the later discussion that it is a judicious choice to use the above new homotopy formulation of the CP.

The above homotopy map encompasses several extra variants. For instance, when $q=1$ and $p \rightarrow \infty$, the above homotopy map, as $u$ varies within the open rectangular box $(0, e)$, reduces to the one proposed by Hotta and Yoshise [16]. When $q=2$ and $p \rightarrow \infty$, the above homotopy map, as $u$ varies within $(0, e)$, is precisely the one studied by Burke and $\mathrm{Xu}[2,3,4]$, and Qi and Sun [26].

It is not difficult to see that if $H(u, x, y)=0$ then $(x, y)$ is a solution to the CP; Conversely, if $(x, y)$ is a solution to the CP, then $(0, x, y)$ is a solution to the equation $H(u, x, y)=0$. Thus, a CP can be solved by locating a solution to the nonlinear equation $H(u, x, y)=0$. The most widely used continuation method for solving this equation is the path-following algorithm that traces certain continuation trajectory leading to the solution set. We do not study such an numerical algorithm in this paper. The purpose here is to establish a theoretical basis for constructing a new non-interior-point path-following algorithm. Such a method can be used to solve a class of problems that is broader than those to which most existing path-following methods can be applied. Given $(a, b, c) \in R_{++}^{n} \times R^{2 n}$, we consider the following system

$$
H(u, x, y)=\theta(a, b, c)
$$

where $\theta \in(0,1]$. Denote by $\bar{Z}=\{\theta(a, b, c): \theta \in(0,1]\}$. In section 4 , we show that the following set

$$
H^{-1}(\bar{Z})=\left\{(u, x, y) \in R_{++}^{n} \times R^{2 n}: H(u, x, y)=\theta(a, b, c), \theta \in(0,1]\right\}
$$

forms a unique, continuous curve leading to a solution of the CP under certain mild conditions. We now give two basic results that will be used later. The first result below gives an equivalent formulation of the system (2.3). This result plays a critical role in the analysis throughout the paper. For the given $(a, b, c) \in R_{++}^{n} \times R^{2 n}$, we define the map $\mathcal{Y}: R^{n} \times(0,1] \rightarrow R^{n}$ by

$$
\begin{aligned}
\mathcal{Y}(x, \theta):=x & +f(x)+\theta^{p} \operatorname{diag}\left(a^{p}\right) x+\theta c \\
& +\sqrt{\left[x-\left(f(x)+\theta^{p} \operatorname{diag}\left(a^{p}\right) x+\theta c\right)\right]^{2}+4 \theta^{q} a^{q}}-\theta b .
\end{aligned}
$$


LEMMA 2.1. The solutions of $\mathcal{Y}(x, \theta)=0$ are in one-to-one correspondence to that of $H(u, x, y)=\theta(a, b, c)$. Specifically, for the given scalar $\theta \in(0,1]$, if $(u, x, y)$ is a solution to the system $H(u, x, y)=\theta(a, b, c)$, then $x$ is a solution to the equation $\mathcal{Y}(x, \theta)=0 ;$ Conversely, if $x$ is a solution to the equation $\mathcal{Y}(x, \theta)=0$, then $(u, x, y)$, where $u=\theta a$ and $y=f(x)+\operatorname{diag}\left\{u^{p}\right\} x+\theta c$, is a solution to the system $H(u, x, y)=$ $\theta(a, b, c)$.

Proof. The result is easy to show. Indeed, the equation $H(u, x, y)=\theta(a, b, c)$ is equivalent to the following system.

$$
\begin{gathered}
u=\theta a, \\
x+y-\sqrt{(x-y)^{2}+4 u^{q}}=\theta b, \\
y=f(x)+\operatorname{diag}\left\{u^{p}\right\} x+\theta c .
\end{gathered}
$$

Substituting the first and the third equations into the second one yields $\mathcal{Y}(x, \theta)=0$. $\square$

It is well known (see Lemma 1.1 in [16]) that for every nonnegative number $\mu \geq 0$, a triplet $(\alpha, \beta, \gamma) \in R^{3}$ satisfies

$$
\phi(\mu, \alpha, \beta)=\alpha+\beta-\sqrt{(\alpha-\beta)^{2}+4 \mu}=\gamma
$$

if and only if $(\alpha-\gamma / 2, \beta-\gamma / 2) \geq 0$ and $(\alpha-\gamma / 2)(\beta-\gamma / 2)=\mu \geq 0$. Moreover, if $\mu>0$, then $(\alpha-\gamma / 2, \beta-\gamma / 2)>0$. By this fact, from $(2.5)-(2.7)$, we have the following Lemma.

Lemma 2.2. Let $(a, b, c) \in R_{++}^{n} \times R^{2 n}$ be a fixed vector. Then for any $\theta \in(0,1]$, the vector $(u(\theta), x(\theta), y(\theta))$ is a solution to the system (2.3) if and only if it satisfies the following system:

$$
\begin{gathered}
u(\theta)=\theta a, \\
y(\theta)=f(x(\theta))+\theta^{p} \operatorname{diag}\left(a^{p}\right) x(\theta)+\theta c, \\
x(\theta)-\theta b / 2>0, y(\theta)-\theta b / 2>0, \\
\operatorname{diag}(x(\theta)-\theta b / 2)(y(\theta)-\theta b / 2)=\theta^{q} a^{q} .
\end{gathered}
$$

Remark 2.1. Since $\theta \in(0,1]$ and $f$ is continuous, it follows from (2.8) and (2.9) that a sequence $\left\{\left(u\left(\theta_{k}\right), x\left(\theta_{k}\right), y\left(\theta_{k}\right)\right)\right\}$, where $\theta_{k} \in(0,1]$, is unbounded if and only if $\left\{x\left(\theta_{k}\right)\right\}$ is unbounded. This fact will be frequently used in the later sections.

3. A new proper condition. In this section, we specify a new condition that is used to prove the existence and boundedness of the trajectory in the next section. To understand this condition better, we show first some properties of a semimonotone function. A map $f: R^{n} \rightarrow R^{n}$ is said to be semimonotone if for any distinct vectors $x, y$ in $R^{n}$ with $x \geq y$, there exists a component $i$ such that $x_{i}>y_{i}$ and $f_{i}(x) \geq f_{i}(y)$. It is evident that each $\mathrm{P}_{0}$-function is semimonotone. The 
following result is a generalization of Lemma 1 in Ravindran and Gowda [27]. The proof is similar to the ones in such works as Tseng [30], Gowda and Tawhid [13], and Facchinei and Kanzow [11].

Lemma 3.1. Let $f: R^{n} \rightarrow R^{n}$ be a continuous semimonotone function. Let $\left\{z^{k}\right\}$ be an arbitrary sequence with $\left\|z^{k}\right\| \rightarrow \infty$ and $z^{k} \geq \bar{z}$ for all $k$, where $\bar{z} \in R^{n}$ is a fixed vector. Then there exist a subsequence of $\left\{z^{k}\right\}$, denoted by $\left\{z^{k_{j}}\right\}$, and a fixed index $i_{0}$ such that $z_{i_{0}}^{k_{j}} \rightarrow \infty$ and $f_{i_{0}}\left(z^{k_{j}}\right)$ is bounded from below.

Proof. Passing through a subsequence, we may assume that there exists an index set $I$ such that $z_{i}^{k} \rightarrow \infty$ for all $i \in I$, and $\left\{z_{i}^{k}\right\}$ is bounded for all $i \notin I$. Construct $\left\{y^{k}\right\}$ as follows: $y_{i}^{k}=\bar{z}_{i}$ if $i \in I$ and $y_{i}^{k}=z_{i}^{k}$ if $i \notin I$. Then we have that $z^{k} \neq y^{k}$ and $z^{k} \geq y^{k}$ for all sufficiently large $k$. By the semimonotone property of $f$, for each sufficiently large $k$ there exists at least one index $i$ such that $z_{i}^{k}>y_{i}^{k}$ and $f_{i}\left(z^{k}\right) \geq f_{i}\left(y^{k}\right)$. Thus, there exist an index $i_{0} \in I$ and a subsequence of $\left\{z^{k}\right\}$, denoted by $\left\{z^{k_{j}}\right\}$, such that $z_{i_{0}}^{k_{j}}>y_{i_{0}}^{k_{j}}$ and $f_{i_{0}}\left(z^{k_{j}}\right) \geq f_{i_{0}}\left(y^{k_{j}}\right)$ for all $j$. By the construction, $\left\{y^{k_{j}}\right\}$ is bounded, and so is $\left\{f_{i_{0}}\left(y^{k_{j}}\right)\right\}$. Hence, $\left\{f_{i_{0}}\left(z^{k_{j}}\right)\right\}$ is bounded from below.

Given $(a, b, c) \in R_{++}^{n} \times R^{2 n}$ and $\theta \in(0,1]$. We define a function $\mathcal{F}_{(a, b, c, \theta)}: R^{2 n} \rightarrow$ $R^{2 n}$ as follows:

$$
\mathcal{F}_{(a, b, c, \theta)}(x, y)=\left(\begin{array}{c}
X y \\
y-f(x+\theta b / 2)-\theta^{p} \operatorname{diag}\left(a^{p}\right) x-\theta c
\end{array}\right),
$$

where $X=\operatorname{diag}(x)$. The next property of semimonotone functions is one of the motivation for our new proper condition.

Proposition 3.1. Let $f: R^{n} \rightarrow R^{n}$ be a continuous semimonotone function. Then for any $(a, b, c, \theta) \in R_{++}^{n} \times R^{2 n} \times(0,1]$, the set

$$
\mathcal{F}_{(a, b, c, \theta)}^{-1}(D)=\left\{(x, y) \in R_{++}^{n}: \mathcal{F}_{(a, b, c, \theta)}(x, y) \in D\right\}
$$

is bounded for any compact set $D$ in $R_{+}^{n} \times R^{n}$.

Proof. Assume the contrary that there exist certain $\left(a^{\prime}, b^{\prime}, c^{\prime}, \theta^{\prime}\right) \in R_{++}^{n} \times R^{2 n} \times$ $(0,1]$ and a compact set $D^{\prime} \subseteq R_{+}^{n} \times R^{n}$ such that $\mathcal{F}_{\left(a^{\prime}, b^{\prime}, c^{\prime}, \theta^{\prime}\right)}^{-1}\left(D^{\prime}\right)$ is unbounded. Let $\left\{\left(x^{k}, y^{k}\right)\right\} \subseteq \mathcal{F}_{\left(a^{\prime}, b^{\prime}, c^{\prime}, \theta^{\prime}\right)}^{-1}\left(D^{\prime}\right)$ such that $\left\|\left(x^{k}, y^{k}\right)\right\| \rightarrow \infty$. Notice that

$$
\mathcal{F}_{\left(a^{\prime}, b^{\prime}, c^{\prime}, \theta^{\prime}\right)}\left(x^{k}, y^{k}\right)=\left(\begin{array}{c}
X^{k} y^{k} \\
y^{k}-f\left(x^{k}+\theta^{\prime} b^{\prime} / 2\right)-\left(\theta^{\prime}\right)^{p} \operatorname{diag}\left(\left(a^{\prime}\right)^{p}\right) x^{k}-\theta^{\prime} c^{\prime}
\end{array}\right) \in D^{\prime} .
$$

There is a sequence $\left\{\left(u^{k}, v^{k}\right)\right\} \subseteq D^{\prime}$, where $u^{k} \in R_{+}^{n}$ and $v^{k} \in R^{n}$, such that for all $k$ we have

$$
\begin{gathered}
X^{k} y^{k}=u^{k} \geq 0, \\
y^{k}=f\left(x^{k}+\theta^{\prime} b^{\prime} / 2\right)+\left(\theta^{\prime}\right)^{p} \operatorname{diag}\left(\left(a^{\prime}\right)^{p}\right) x^{k}+\theta^{\prime} c^{\prime}+v^{k} .
\end{gathered}
$$

Since $\left\{\left(u^{k}, v^{k}\right)\right\}$ is bounded and since $\left\|\left(x^{k}, y^{k}\right)\right\| \rightarrow \infty$, by continuity we conclude that the sequence $\left\{x^{k}\right\}$ is unbounded. Thus, we may assume that $\left\|x^{k}\right\| \rightarrow \infty$. Since $x^{k} \in R_{+}^{n}$ for all $k$, passing through a subsequence we may assume that there exists an index set $I$ such that $x_{i}^{k} \rightarrow \infty$ for all $i \in I$, and $\left\{x_{i}^{k}\right\}$ is bounded for all $i \notin I$. Since $x_{i}^{k} \rightarrow \infty$ for all $i \in I$, it follows from (3.2) that $y_{i}^{k} \rightarrow 0$ for all $i \in I$. Hence, from (3.3) we have

$$
f_{i}\left(x^{k}+\theta^{\prime} b^{\prime} / 2\right)=y_{i}^{k}-\left(\theta^{\prime}\right)^{p} \operatorname{diag}\left(\left(a^{\prime}\right)^{p}\right) x_{i}^{k}-\theta^{\prime} c^{\prime}{ }_{i}-v_{i}^{k} \rightarrow-\infty
$$


for all $i \in I$. However, by Lemma 3.1, there exists an index $i \in I$ such that $\left\{f_{i}\left(x^{k}+\right.\right.$ $\left.\left.\theta^{\prime} b^{\prime} / 2\right)\right\}$ is bounded from below. This is a contradiction.

Particularly, Denote by $D_{r}:=\left[0, r v_{1}\right] \times\left[-r v_{2}, r v_{3}\right] \subseteq R_{+}^{n} \times R^{n}$ where $v_{i} \in R_{+}^{n}(i=$ $1,2,3)$ and $r$ is a nonnegative number. We deduce from the above proposition that the set $\mathcal{F}_{(a, b, c, r)}^{-1}\left(D_{r}\right)$ is bounded for any $0<r<\infty$ if $f$ is continuous and semimonotone. Inspired by this observation, we impose the following proper condition on the CP.

CONDITION 3.1. For any given $(a, b, c) \in R_{++}^{n} \times R^{2 n}$ and scalar $\hat{t} \geq 0$, there exists a scalar $1 \geq \theta^{*}>0$ such that

$$
\bigcup_{\theta \in\left(0, \theta^{*}\right]} \mathcal{F}_{(a, b, c, \theta)}^{-1}\left(D_{\theta}\right)
$$

is bounded, where

$$
\mathcal{F}_{(a, b, c, \theta)}^{-1}\left(D_{\theta}\right):=\left\{(x, y) \in R_{++}^{2 n}: \mathcal{F}_{(a, b, c, \theta)}(x, y) \in D_{\theta}\right\}
$$

and

$$
D_{\theta}:=\left[0, \theta a^{q}\right] \times[-\theta \hat{t} e, \theta \hat{t} e] \subseteq R_{+}^{n} \times R^{n} .
$$

Notice that for a fixed $\bar{\theta} \in(0,1)$, the above set $D_{\theta} \subseteq D_{\bar{\theta}}:=[0, \bar{\theta} e] \times[-\bar{\theta} e, \bar{\theta} e]$ for all sufficiently small $\theta$. Thus, we can see that Condition 3.1 holds if the following condition is satisfied.

Condition 3.2. For any given $(a, b, c) \in R_{++}^{n} \times R^{2 n}$, there exists a scalar $1>$ $\bar{\theta}>0$ such that

$$
\bigcup_{\theta \in(0, \bar{\theta}]} \mathcal{F}_{(a, b, c, \theta)}^{-1}\left(D_{\bar{\theta}}\right)
$$

is bounded, where $D_{\bar{\theta}}=[0, \bar{\theta} e] \times[-\bar{\theta} e, \bar{\theta} e]$ and

$$
\mathcal{F}_{(a, b, c, \theta)}^{-1}\left(D_{\bar{\theta}}\right)=\left\{(x, y) \in R_{++}^{n}: \mathcal{F}_{(a, b, c, \theta)}(x, y) \in D_{\bar{\theta}}\right\}
$$

The above Condition 3.1 may seem to be a little unusual at first glance. As we will see subsequently, this condition is actually quite weak. A prominent feature of Condition 3.1 is that it may hold even when the solution set of the $\mathrm{CP}$ is unbounded or the strict feasibility condition fails to hold. Specifically, for $\mathrm{P}_{0}$-complementarity problems, we will show that the previously known conditions such as Condition 1.1, Condition 1.2, the nonemptyness and boundedness assumption of the solution set (in particular, the $\mathrm{P}_{0}$ together with $\mathrm{R}_{0}$ property), and Condition 1.5 in [19] all imply the above Condition 3.1. However, the converse is not true (Theorem 3.1). Before we prove this fact, we list some helpful results. The following result is easy to prove by using the compactness of $S$ and continuity of $f$. Its proof is omitted.

Lemma 3.2. Let $S$ be a compact set in $R^{n}$ and $(a, b, c) \in R_{++}^{n} \times R^{2 n}$ be a fixed triplet. Let $f$ be a continuous function from $R^{n}$ into itself.

(i) Let

$$
G(x):=x+f(x)-\sqrt{(x-f(x))^{2}} .
$$


Define $\bar{G}: R^{n} \times(0,1] \times R_{+}^{n} \times R^{n} \rightarrow R^{n}$ by

$$
\begin{aligned}
& \bar{G}(x, \theta, w, v)=x+f(x)+\theta^{p} \operatorname{diag}\left(a^{p}\right)(x-\theta b / 2)+\theta(c+b / 2)+v \\
& -\sqrt{\left[x-\left(f(x)+\theta^{p} \operatorname{diag}\left(a^{p}\right)(x-\theta b / 2)+\theta(c+b / 2)+v\right)\right]^{2}+4 w}-\theta b .
\end{aligned}
$$

Then for any $\delta>0$, there exists a scalar $\bar{\theta} \in(0,1]$ such that for all $\theta \in(0, \bar{\theta}]$ and $(w, v) \in[0, \bar{\theta} e] \times[-\bar{\theta} e, \bar{\theta} e] \subseteq R_{+}^{n} \times R^{n}$, we have

$$
\sup _{x \in S}\|\bar{G}(x, \theta, w, v)-G(x)\|<\delta .
$$

(ii) Given any $\hat{\theta} \in(0,1)$, then for any $\delta>0$ there exists a sufficiently small scalar $\beta>0$ such that

$$
\sup _{x \in S}\|\mathcal{Y}(x, \theta)-\mathcal{Y}(x, \hat{\theta})\|<\delta \text { for all } \theta \text { such that }|\theta-\hat{\theta}|<\beta,
$$

where $\mathcal{Y}(x, \theta)$ is defined by $(2.4)$.

The next result, which was pointed out by Gowda and Tawhid [13], is very useful for the subsequent analysis.

LemmA 3.3. Let $\Phi(x, v)=x+f(x)-\sqrt{(x-f(x))^{2}+v^{2}}$ where $v \in R^{n}$.

(i) If $f$ is a $P_{0}$-function, then $\Phi(x, v)$ is a $P_{0}$-function in $x$. Moreover, if $v^{2} \in$ $R_{++}^{n}$, then $\Phi(x, v)$ is a P-function in $x$.

(ii) If $f$ is a P-function, then $\Phi(x, v)$ is a P-function in $x$.

The following upper-semicontinuity property of a $\mathrm{P}_{0}$-function is due to Ravindran and Gowda [27].

Lemma 3.4. Let $g: R^{n} \rightarrow R^{n}$ be a $P_{0}$-function. Suppose that $g^{-1}(0)$ is nonempty and compact. Then for any given $\varepsilon>0$, there exists a scalar $\gamma>0$ such that for any $P_{0}$-function $h$ with

$$
\sup _{\bar{\Omega}}\|h(x)-g(x)\|<\gamma,
$$

we have

$$
\emptyset \neq h^{-1}(0) \subseteq g^{-1}(0)+\varepsilon B
$$

where $B$ denotes the open unit ball in $R^{n}$ and $\bar{\Omega}$ is the closure of the set $\Omega=g^{-1}(0)+$ $\varepsilon B$.

We now show that several well-known existing conditions used in the literature of interior-point and non-interior-point methods imply Condition 3.1. However, the converse is not true since Condition 3.1 may hold for $\mathrm{P}_{0}$-CPs in the absence of the strict feasibility condition.

THEOREM 3.1. Let $f$ be a $P_{0}$-function. If one of the following condition holds,

(i) Condition 1.1,

(ii) Condition 1.2,

(iii) Condition 1.5 in Kojima et al. [19],

(iv) the solution set of the $C P$ is nonempty and bounded,

(v) $f$ is a $P_{0}$ and $R_{0}$ function $[1,6]$,

then Condition 3.1 holds. However, the converse is not true, i.e., Condition 3.1 does not imply any one of the above conditions. 
Proof. The implication (ii) $\Rightarrow$ (i) is pointed out in $[16,26]$. It is easy to verify that (i) $\Rightarrow$ (iv). In fact, if (i) holds, Hotta and Yoshise [16] showed that their noninterior-point trajectory exists and a subtrajectory is bounded, and hence each of the accumulation points of the subtrajectory is a solution to the CP. Hence, the solution set of the CP is not empty. We further demonstrate that it is bounded. Indeed, by Condition 1.1, there is a point $x^{0}>0$ such that $f\left(x^{0}\right)>0$. It follows from Lemma 2.1 in [16] that $R_{-}^{n} \times R_{+}^{n} \subseteq V\left(R_{++}^{n} \times R^{2 n}\right)$. Thus by Condition 1.1 again, the set $U^{-1}(D)$ is bounded for every compact subset $D$ of $R_{+}^{n} \times R_{-}^{n} \times R_{+}^{n}$. In particular, set

$$
D:=\{(0,0,0)\} \subseteq R_{+}^{n} \times R_{-}^{n} \times R_{+}^{n} .
$$

Then the set $U^{-1}(0)$ is bounded. The set $U^{-1}(0)$ coincides with the solution set of the CP. Hence (i) $\Rightarrow$ (iv).

By the proof similar to the above, we can show that (iii) $\Rightarrow$ (iv). The implication of $(\mathrm{v}) \Rightarrow(\mathrm{iv})$ is a known result.

Therefore, to show each condition of (i)-(v) implies Condition 3.1, it is sufficient to prove that (iv) implies Condition 3.1. Indeed, assume that $f$ is a $\mathrm{P}_{0}$-function and the solution set of the CP is nonempty and bounded. We show that Condition 3.2 holds (and hence Condition 3.1 holds). Let $G: R^{n} \rightarrow R^{n}$ be given by

$$
G(x):=x+f(x)-\sqrt{(x-f(x))^{2}},
$$

which is a $\mathrm{P}_{0}$-function (Lemma 3.3). Since $G^{-1}(0)=\left\{x \in R^{n}: G(x)=0\right\}$ coincides with the solution set of the CP, by the assumption, the set $G^{-1}(0)$ is nonempty and bounded, in fact, a compact set by the continuity of $f$. For any scalar $\varepsilon>0$, by Lemma 3.4, there is a scalar $\delta>0$ such that for any $\mathrm{P}_{0}$-function $h: R^{n} \rightarrow R^{n}$ with

$$
\sup _{x \in \bar{\Omega}}\|h(x)-G(x)\|<\delta
$$

where $\Omega=G^{-1}(0)+\varepsilon B$, then

$$
0 \neq h^{-1}(0) \subseteq G^{-1}(0)+\varepsilon B .
$$

Let $(a, b, c)$ be a fixed triplet in $R_{++}^{n} \times R^{2 n}$, and let $\bar{G}(x, \theta, w, v)$ be given as in Lemma 3.2 , where $\theta \in(0,1]$ and $(w, v) \in R_{+}^{n} \times R^{n}$. Clearly, the function

$$
f(x)+\operatorname{diag}\left(\theta^{p} a^{p}\right)(x-\theta b / 2)+\theta(c+b / 2)+v
$$

is a P-function in $x$. Since $w \in R_{+}^{n}$, it follows from (ii) of Lemma 3.3 that $\bar{G}(x, \theta, w, v)$ is a P-function in $x$. By (i) of Lemma 3.2, there exists a sufficiently small number $\bar{\theta} \in(0,1)$ such that for all $\theta \in(0, \bar{\theta}]$ and $(w, v) \in[0, \bar{\theta} e] \times[-\bar{\theta} e, \bar{\theta} e]$, we have

$$
\sup _{x \in \bar{\Omega}}\|\bar{G}(x, \theta, w, v)-G(x)\|<\delta .
$$

Thus setting $h(x):=\bar{G}(x, \theta, w, v)$ in (3.4), we have from (3.5) that

$$
\emptyset \neq \bar{G}_{(\theta, w, v)}^{-1}(0) \subseteq G^{-1}(0)+\varepsilon B
$$

for all $\theta \in(0, \bar{\theta}]$ and $(w, v) \in[0, \bar{\theta}] \times[-\bar{\theta} e, \bar{\theta} e]$, where

$$
\bar{G}_{(\theta, w, v)}^{-1}(0)=\left\{x \in R^{n}: \bar{G}(x, \theta, w, v)=0\right\} .
$$


Hence,

$$
\bigcup_{(\theta, w, v) \in(0, \bar{\theta}] \times D_{\bar{\theta}}} \bar{G}_{(\theta, w, v)}^{-1}(0) \subseteq G^{-1}(0)+\varepsilon B,
$$

where $D_{\bar{\theta}}=[0, \bar{\theta} e] \times[-\bar{\theta} e, \bar{\theta} e]$. On the other hand, it is easy to verify that

$$
\bar{G}(x, \theta, w, v)=0, \theta \in(0, \bar{\theta}],(w, v) \in D_{\bar{\theta}}
$$

if and only if

$$
\begin{aligned}
& x-\theta b / 2 \geq 0, y-\theta b / 2 \geq 0, \\
& \operatorname{diag}(x-\theta b / 2)(y-\theta b / 2)=w, \\
& y-\theta b / 2-f(x)-\theta^{p} \operatorname{diag}\left(a^{p}\right)(x-\theta b / 2)-\theta c=v, \\
& \theta \in(0, \bar{\theta}], \quad(w, v) \in D_{\bar{\theta}} .
\end{aligned}
$$

Denote by $\bar{x}=x-\theta b / 2$ and $\bar{y}=y-\theta b / 2$. The above system can be rewritten as

$$
\mathcal{F}_{(a, b, c, \theta)}(\bar{x}, \bar{y}) \in D_{\bar{\theta}},(\bar{x}, \bar{y}) \geq 0, \theta \in(0, \bar{\theta}],
$$

where $\mathcal{F}_{(a, b, c, \theta)}$ is defined by (3.1). Denote

$$
\mathcal{F}_{(a, b, c, \theta)}^{-1}\left(D_{\bar{\theta}}\right)=\left\{(u, v) \in R_{++}^{2 n}: \mathcal{F}_{(a, b, c, \theta)}(u, v) \in D_{\bar{\theta}}\right\} .
$$

Then from the above discussion, we deduce that

$$
\begin{aligned}
& \left\{x \in R^{n}: x=\bar{x}+\theta b / 2,(\bar{x}, \bar{y}) \in \mathcal{F}_{(a, b, c, \theta)}^{-1}\left(D_{\bar{\theta}}\right), \theta \in(0, \bar{\theta}]\right\} \\
& \subseteq\left\{x \in R^{n}: x=\bar{x}+\theta b / 2, \mathcal{F}_{(a, b, c, \theta)}(\bar{x}, \bar{y}) \in D_{\bar{\theta}},(\bar{x}, \bar{y}) \geq 0, \theta \in(0, \bar{\theta}]\right\} \\
& =\bigcup_{(\theta, w, v) \in(0, \bar{\theta}] \times D_{\bar{\theta}}} \bar{G}_{(\theta, w, v)}^{-1}(0) \\
& \subseteq G^{-1}(0)+\varepsilon B .
\end{aligned}
$$

Since $G^{-1}(0)+\varepsilon B$ is bounded, we deduce from the above that

$$
\bigcup_{\theta \in(0, \bar{\theta}]} \mathcal{F}_{(a, b, c, \theta)}^{-1}\left(D_{\bar{\theta}}\right)
$$

is bounded. Hence, Condition 3.2 is satisfied, and hence Condition 3.1 holds.

Since each of the conditions listed in the theorem implies the existence of a strictly feasible point, to show that Condition 3.1 does not imply each of these conditions, it suffices to prove that Condition 3.1 may hold even when there is no strictly feasible point. Now consider, in $R^{2}$, the following example:

$$
f(x)=\left(\begin{array}{ll}
0 & 0 \\
0 & 2
\end{array}\right)\left(\begin{array}{l}
x_{1} \\
x_{2}
\end{array}\right)+\left(\begin{array}{c}
0 \\
-1
\end{array}\right)=\left(\begin{array}{c}
0 \\
2 x_{2}-1
\end{array}\right),
$$

which is a $\mathrm{P}_{0}$-function. Clearly, $f$ has no strictly feasible point, and the corresponding complementarity problem has an unbounded solution set. However, this example does satisfy Condition 3.1. Indeed, choose $p \in(0,1]$ and $q \in[1, \infty]$ and let $(a, b, c) \in R_{++}^{2} \times R^{2} \times R^{2}$ be a fixed vector. We show for each scalar $0<\theta^{*}<1$ that the set $\cup_{\theta \in\left(0, \theta^{*}\right]} \mathcal{F}_{(a, b, c, \theta)}^{-1}\left(D_{\theta}\right)$ is bounded set, where all symbols are defined as 
in Condition 3.1. Assume that $\left\{\left(x^{k}, y^{k}\right)\right\}$ is an arbitrary sequence contained in the set. Then, $\left(x^{k}, y^{k}\right)>0$ and for each $\left(x^{k}, y^{k}\right)$ there is a scalar $\theta_{k} \in\left(0, \theta^{*}\right]$ such that $\mathcal{F}_{\left(a, b, c, \theta_{k}\right)}\left(x^{k}, y^{k}\right) \in D_{\theta_{k}}$. By the definitions of $D_{\theta_{k}}$ and $\mathcal{F}_{(a, b, c, \theta)}$, there exist two vectors $d^{k} \in\left[0, a^{q}\right]$ and $\bar{d}^{k} \in[-\hat{t} e, \hat{t} e]$, such that

$$
\begin{aligned}
& X^{k} y^{k}=\theta_{k} d^{k} \in\left[0, \theta_{k} a^{q}\right] \\
& y^{k}-f\left(x^{k}+\theta_{k} b / 2\right)-\theta_{k}^{p} \operatorname{diag}\left(a^{p}\right) x^{k}-\theta_{k} c=\theta_{k} \bar{d}^{k} \in \theta_{k}[-\hat{t} e, \hat{t} e]
\end{aligned}
$$

where $X^{k}=\operatorname{diag}\left(x^{k}\right)$. For this example, the second equation above can be rewritten as

$$
\begin{gathered}
y_{1}^{k}=\left(\theta_{k} a_{1}\right)^{p} x_{1}^{k}+\theta_{k} c_{1}+\theta_{k} \bar{d}_{1}^{k}, \\
y_{2}^{k}=2\left(x_{2}^{k}+\theta_{k} b_{2} / 2\right)-1+\left(\theta_{k} a_{2}\right)^{p} x_{2}^{k}+\theta_{k} c_{2}+\theta_{k} \bar{d}_{2}^{k} .
\end{gathered}
$$

Thus, from $X^{k} y^{k}=\theta_{k} d^{k}$, we have

$$
\theta_{k} d_{1}^{k}=x_{1}^{k} y_{1}^{k}=\left(\theta_{k} a_{1}\right)^{p}\left(x_{1}^{k}\right)^{2}+\theta_{k} x_{1}^{k} c_{1}+\theta_{k} x_{1}^{k} \bar{d}_{1}^{k},
$$

i.e.,

$$
\theta_{k}^{1-p} d_{1}^{k}=a_{1}^{p}\left(x_{1}^{k}\right)^{2}+\theta_{k}^{1-p}\left(c_{1}+\bar{d}_{1}^{k}\right) x_{1}^{k}
$$

and

$$
\theta_{k} d_{2}^{k}=x_{2}^{k} y_{2}^{k}=\left(2+\left(\theta_{k} a_{2}\right)^{p}\right)\left(x_{2}^{k}\right)^{2}+\left[\theta_{k}\left(b_{2}+c_{2}+\bar{d}_{2}^{k}\right)-1\right] x_{2}^{k} .
$$

From the above two relations, we conclude that the sequence $\left\{x^{k}\right\}$ is bounded, and by continuity so is $\left\{y^{k}\right\}$. Therefore, the set $\cup_{\theta \in\left(0, \theta^{*}\right]} \mathcal{F}_{(a, b, c, \theta)}^{-1}\left(D_{\theta}\right)$ is bounded, i.e., Condition 3.1 is satisfied.

4. Existence and boundedness of the trajectory. The purpose of this section is to show the existence and the boundedness of the proposed continuation trajectory for $\mathrm{P}_{0}$-CPs under Condition 3.1. To begin with, we recall a useful result on the degree of a continuous function. Let $\Omega$ be a bounded open set in $R^{n}$. The symbols $\bar{\Omega}$ and $\partial \Omega$ denote the closure and boundary of $\Omega$, respectively. Let $h$ be a continuous function from $\bar{\Omega}$ into $R^{n}$. For any vector $y \in R^{n}$ such that $y \notin h(\partial \Omega)$, then the degree of $h$ at $y$ with respect to $\Omega$ is defined by $\operatorname{deg}(h, \Omega, y)$. The following result can be found in Lloyd [22].

LEMmA 4.1. (i) If $h$ is injective on $R^{n}$, then for any $y \in h(\Omega),|\operatorname{deg}(h, \Omega, y)|=1$.

(ii) If $\operatorname{deg}(h, \Omega, y) \neq 0$, then the equation $h(x)=y$ has a solution in $\Omega$.

(iii) Let $g$ be a continuous function from $\bar{\Omega} \rightarrow R^{n}$. Let

$$
\mathcal{H}(x, t)=\operatorname{tg}(x)+(1-t) h(x), 0 \leq t \leq 1 .
$$

If $y \notin\{\mathcal{H}(x, t): x \in \partial \Omega, t \in[0,1]\}$, then $\operatorname{deg}(g, \Omega, y)=\operatorname{deg}(h, \Omega, y)$.

We are ready to prove a general and essential result.

TheOREM 4.1. Let $(a, b, c)$ be a fixed vector in $R_{++}^{n} \times R^{2 n}$. Let $f: R^{n} \rightarrow R^{n}$ be a continuous semimonotone function.

(i) For each $\theta \in(0,1]$, the system (2.3) has a solution. 
(ii) If Condition 3.1 holds, then the set

$$
\left\{(u, x, y) \in \mathcal{T}_{\theta}: \theta \in(0,1]\right\}:=\bigcup_{\theta \in(0,1]} \mathcal{T}_{\theta}
$$

is bounded, where

$$
\mathcal{T}_{\theta}:=\{(u, x, y): H(u, x, y)=\theta(a, b, c)\} .
$$

Proof. Let $f$ be a continuous semimonotone function. We show the result by contradiction. Assume that there is a scalar $\hat{\theta} \in(0,1]$ such that the system $(2.3)$ has no solution.

Let $\mathcal{H}: R^{n} \times[0,1] \rightarrow R^{n}$ be defined by

$$
\mathcal{H}(x, t)=t(x-\hat{\theta} b / 2)+(1-t) \mathcal{Y}(x, \hat{\theta}), t \in[0,1],
$$

where $\mathcal{Y}$ is given by (2.4). We first show that the set

$$
\mathcal{S}=\left\{x \in R^{n}: \mathcal{H}(x, t)=0 \text { for some } t \in[0,1]\right\}
$$

is unbounded. Indeed, assume the contrary that $\mathcal{S}$ is bounded. Then for any fixed $\varepsilon>0$, the set $D:=(\{\hat{\theta} b / 2\} \cup \mathcal{S})+\varepsilon B$ is a bounded open set in $R^{n}$, where $B$ is the open unit ball in $R^{n}$. Clearly, the intersection of $\mathcal{S}$ and the boundary of $D$ is empty, i.e., for all $x \in \partial D, \mathcal{H}(x, t) \neq 0$ for all $t \in[0,1]$. Therefore,

$$
|\operatorname{deg}(\mathcal{Y}(\cdot, \hat{\theta}), D, 0)|=|\operatorname{deg}(g, D, 0)|=1,
$$

where $g(x):=x-\hat{\theta} b / 2$. The first equation above follows from (iii) of Lemma 4.1, and the second equation follows from (i) of Lemma 4.1 since $g$ is an injective mapping. Thus it follows from (ii) of Lemma 4.1 that the equation $\mathcal{Y}(x, \hat{\theta})=0$ has a solution (in $D)$. Thus by Lemma $2.1, H(u, x, y)=\hat{\theta}(a, b, c)$ has a solution. This contradicts our assumption at the beginning of the proof. Therefore, the set $\mathcal{S}$ is unbounded.

Since $\mathcal{S}$ is unbounded, there is a sequence $\left\{x^{k}\right\}$ contained in $\mathcal{S}$ such that $\left\|x^{k}\right\| \rightarrow$ $\infty$. We now show that $\left\{x^{k}\right\}$ satisfies the relations

$$
x^{k}-\hat{\theta} b / 2>0
$$

and

$$
f\left(x^{k}\right) \leq-\hat{\theta}^{p} \operatorname{diag}\left(a^{p}\right) x^{k}+2 \hat{\theta}^{q}\left[\operatorname{diag}\left(x^{k}-\hat{\theta} b / 2\right)\right]^{-1} a^{q}+\hat{\theta}(b / 2-c)
$$

for all sufficiently large $k$. Indeed, since $\left\|x^{k}\right\| \rightarrow \infty$, there is a $k_{0}>0$ such that $\left\|x^{k}-\hat{\theta} b / 2\right\|>0$ for all $k>k_{0}$. Since $x^{k} \in \mathcal{S}$, by the definition of $\mathcal{S}$, there is a scalar $t^{k} \in[0,1]$ such that

$$
\mathcal{H}\left(x^{k}, t^{k}\right)=t^{k}\left(x^{k}-\hat{\theta} b / 2\right)+\left(1-t^{k}\right) \mathcal{Y}\left(x^{k}, \hat{\theta}\right)=0 .
$$

Since $x^{k} \neq \hat{\theta} b / 2$ for all $k>k_{0}$, we deduce from the above that $t^{k} \neq 1$ for all $k>k_{0}$. Since the system $(2.3)$ has no solution (by the assumption), i.e., $\mathcal{Y}(x, \hat{\theta}) \neq 0$ for all $x \in R^{n}$ (Lemma 2.1), it follows from the above equation that $t^{k} \neq 0$. Therefore, we have that $t^{k} \in(0,1)$ for all $k>k_{0}$. The above equation can be written as

$$
\begin{aligned}
& t^{k}\left(x^{k}-\hat{\theta} b / 2\right)+\left(1-t^{k}\right)\left[\left(x^{k}-\hat{\theta} b / 2\right)+\left(y^{k}-\hat{\theta} b / 2\right)\right] \\
& -\left(1-t^{k}\right) \sqrt{\left[\left(x^{k}-\hat{\theta} b / 2\right)-\left(y^{k}-\hat{\theta} b / 2\right)\right]^{2}+4 \hat{\theta}^{q} a^{q}}=0,
\end{aligned}
$$


where

$$
y^{k}=f\left(x^{k}\right)+\hat{\theta}^{p} \operatorname{diag}\left(a^{p}\right) x^{k}+\hat{\theta} c .
$$

Denote by

$$
\hat{x}^{k}=x^{k}-\hat{\theta} b / 2, \quad \hat{y}^{k}=y^{k}-\hat{\theta} b / 2 .
$$

The above equation can be further written as

$$
\hat{x}^{k}+\left(1-t^{k}\right) \hat{y}^{k}=\left(1-t^{k}\right) \sqrt{\left(\hat{x}^{k}-\hat{y}^{k}\right)^{2}+4 \hat{\theta}^{q} a^{q}} .
$$

Squaring both sides of the above equation and simplifying (all the algebraic operations are performed componentwise), we obtain

$$
t^{k}\left(2-t^{k}\right)\left(\hat{x}^{k}\right)^{2}+2\left(1-t^{k}\right)\left(2-t^{k}\right) \hat{X}^{k} \hat{y}^{k}=4\left(1-t^{k}\right)^{2} \hat{\theta}^{q} a^{q},
$$

where $\hat{X}^{k}=\operatorname{diag}\left(\hat{x}^{k}\right)$. It follows from the above that $\hat{x}_{i}^{k} \neq 0$ for all $i=1,2, \ldots, n$. Multiplying both sides of the above by $\left(\hat{X}^{k}\right)^{-1}$ and dividing both sides by $2(1-$ $\left.t^{k}\right)\left(2-t^{k}\right)$ yield

$$
\hat{y}^{k}=-\frac{t^{k}}{2\left(1-t^{k}\right)} \hat{x}^{k}+\frac{2\left(1-t^{k}\right) \hat{\theta}^{q}}{2-t^{k}}\left(\hat{X}^{k}\right)^{-1} a^{q} .
$$

Thus, we have

$$
\hat{x}^{k}+\left(1-t^{k}\right) \hat{y}^{k}=\left(1-\frac{t^{k}}{2}\right) \hat{x}^{k}+\frac{2\left(1-t^{k}\right)^{2} \hat{\theta}^{q}}{2-t^{k}}\left(\hat{X}^{k}\right)^{-1} a^{q} .
$$

If $\hat{x}_{i}^{k} \leq 0$ for some $i$, then we have from the above that $\hat{x}_{i}^{k}+\left(1-t^{k}\right) \hat{y}_{i}^{k} \leq 0$ which contradicts the right-hand side of (4.2). Thus $\left\{\hat{x}^{k}\right\} \subseteq R_{++}^{n}$ and

$$
\hat{y}^{k} \leq \frac{2\left(1-t^{k}\right) \hat{\theta}^{q}}{2-t^{k}}\left(\hat{X}^{k}\right)^{-1} a^{q} \leq 2 \hat{\theta}^{q}\left(\hat{X}^{k}\right)^{-1} a^{q}
$$

That is, $x^{k}-\hat{\theta} b / 2>0$ and

$$
f\left(x^{k}\right) \leq-\hat{\theta}^{p} \operatorname{diag}\left(a^{p}\right) x^{k}+2 \hat{\theta}^{q}\left[\operatorname{diag}\left(x^{k}-\hat{\theta} b / 2\right)\right]^{-1} a^{q}+\hat{\theta}(b / 2-c)
$$

for all $k>k_{0}$. Passing through a subsequence, we may suppose that there is an index set $I$ such that $x_{i}^{k} \rightarrow \infty$ for all $i \in I$ and $\left\{x_{i}^{k}\right\}$ is bounded for $i \notin I$. It follows from (4.3) that $f_{i}\left(x^{k}\right) \rightarrow-\infty$ for all $i \in I$. This contradicts the consequence of Lemma 3.1 which states that there exists an index $i \in I$ such that $f_{i}\left(x^{k}\right)$ is bounded from below. Hence, Item (i) of the theorem is shown.

We now prove Item (ii) of the theorem, i.e., the boundedness of the set (4.1). Assume the contrary that the set $\left\{(u, x, y) \in \mathcal{T}_{\theta}: \theta \in(0,1]\right\}$ is unbounded, i.e., there exists an unbounded sequence $\left\{\left(u\left(\theta_{k}\right), x\left(\theta_{k}\right), y\left(\theta_{k}\right)\right)\right\}$ contained in the set, where $0<\theta_{k} \leq 1$. Thus the sequence $\left\{x\left(\theta_{k}\right)\right\}$ is unbounded (Remark 2.1). Without loss of generality, we assume that $\left\|x\left(\theta_{k}\right)\right\| \rightarrow \infty$ as $k \rightarrow \infty$. Note that $\left\{\left(u\left(\theta_{k}\right), x\left(\theta_{k}\right), y\left(\theta_{k}\right)\right)\right\}$ satisfy the system (2.8)-(2.11) where $\theta$ is replaced by $\theta_{k}$. By the unboundedness of $\left\{x\left(\theta_{k}\right)\right\}$ and $x\left(\theta_{k}\right) \geq \hat{\theta} b / 2$, it follows from Lemma 3.1 that there exist a subsequence 
of $\left\{x\left(\theta_{k}\right)\right\}$ denoted also by $\left\{x\left(\theta_{k}\right)\right\}$ and an index $m$ such that $x_{m}\left(\theta_{k}\right) \rightarrow \infty$ and $f_{m}\left(x\left(\theta_{k}\right)\right)$ is bounded from below. From (2.11), we have

$$
y_{m}\left(\theta_{k}\right)-\theta_{k} b_{m} / 2=\frac{\theta_{k}^{q} a_{m}^{q}}{x_{m}\left(\theta_{k}\right)-\theta_{k} b_{m} / 2},
$$

and by using (2.9) we have

$$
f_{m}\left(x\left(\theta_{k}\right)\right)=\theta_{k} b_{m} / 2+\frac{\theta_{k}^{q} a_{m}^{q}}{x_{m}\left(\theta_{k}\right)-\theta_{k} b_{m} / 2}-\theta_{k} c_{m}-\left(\theta_{k} a_{m}\right)^{p} x_{m}\left(\theta_{k}\right) .
$$

Since $x_{m}\left(\theta_{k}\right) \rightarrow \infty$ and $f_{m}\left(x\left(\theta_{k}\right)\right)$ is bounded from below, we deduce from the above that $\theta_{k}^{p} \rightarrow 0$, and thus $\theta_{k} \rightarrow 0$. Denote

$$
\hat{x}\left(\theta_{k}\right)=x\left(\theta_{k}\right)-\theta_{k} b / 2, \quad \hat{y}\left(\theta_{k}\right)=y\left(\theta_{k}\right)-\theta_{k} b / 2 .
$$

By (2.10) and (2.11), we have

$$
\left(\hat{x}\left(\theta_{k}\right), \hat{y}\left(\theta_{k}\right)\right)>0, \quad \hat{X}\left(\theta_{k}\right) \hat{x}\left(\theta_{k}\right)=\theta_{k}^{q} a^{q} .
$$

By using (2.9) again, we have

$$
\begin{aligned}
& \hat{y}\left(\theta_{k}\right)-f\left(\hat{x}\left(\theta_{k}\right)+\theta_{k} b / 2\right)-\theta_{k}^{p} \operatorname{diag}\left(a^{p}\right) \hat{x}\left(\theta_{k}\right)-\theta_{k} c \\
& =y\left(\theta_{k}\right)-\theta_{k} b / 2-f\left(x\left(\theta_{k}\right)\right)-\theta_{k}^{p} \operatorname{diag}\left(a^{p}\right) x\left(\theta_{k}\right)+\theta_{k}^{p} \operatorname{diag}\left(a^{p}\right)\left(\theta_{k} b / 2\right)-\theta_{k} c \\
& =-\theta_{k} b / 2+\theta_{k}^{p} \operatorname{diag}\left(a^{p}\right)\left(\theta_{k} b / 2\right) \\
& =\theta_{k}\left[-b / 2+\theta_{k}^{p} \operatorname{diag}\left(a^{p}\right) b / 2\right] .
\end{aligned}
$$

Let $\hat{t}=\|-b / 2\|_{\infty}+\left\|\operatorname{diag}\left(a^{p}\right) b / 2\right\|_{\infty}$. Then, for any $\theta^{k} \in(0,1]$, we have $-\hat{t} e \leq$ $-b / 2+\theta_{k}^{p} \operatorname{diag}\left(a^{p}\right) b / 2 \leq \hat{t} e$. Therefore,

$$
\begin{gathered}
\theta_{k}^{q} a^{q}=\theta_{k}\left(\theta_{k}^{q-1} a^{q}\right) \in\left[0, \theta_{k} a^{q}\right], \\
\theta_{k}\left(-b / 2+\theta_{k}^{p} \operatorname{diag}\left(a^{p}\right) b / 2\right) \in \theta_{k}[-\hat{t} e, \hat{t} e] .
\end{gathered}
$$

Therefore,

$$
\begin{aligned}
\mathcal{F}_{\left(a, b, c, \theta_{k}\right)}\left(\hat{x}\left(\theta_{k}\right), \hat{y}\left(\theta_{k}\right)\right) & =\left(\begin{array}{c}
\hat{X}\left(\theta_{k}\right) \hat{y}\left(\theta_{k}\right) \\
\hat{y}\left(\theta_{k}\right)-f\left(\hat{x}\left(\theta_{k}\right)+\theta_{k} b / 2\right)-\theta_{k}^{p} \operatorname{diag}\left(a^{p}\right) \hat{x}\left(\theta_{k}\right)-\theta_{k} c
\end{array}\right) \\
& \in\left[0, \theta_{k} a^{q}\right] \times \theta_{k}[-\hat{t} e, \hat{t} e]=: D_{\theta_{k}}
\end{aligned}
$$

for all $\theta_{k} \in(0,1]$. Thar is, $\left(\hat{x}\left(\theta_{k}\right), \hat{y}\left(\theta_{k}\right)\right) \in \mathcal{F}_{\left(a, b, c, \theta_{k}\right)}^{-1}\left(D_{\theta_{k}}\right)$ for all $\theta_{k} \in(0,1]$. Hence, for any $1 \geq \theta^{*}>0$, the sequence

$$
\left\{\left(\hat{x}\left(\theta_{k}\right), \hat{y}\left(\theta_{k}\right)\right): \theta_{k} \in\left(0, \theta^{*}\right]\right\} \subseteq \bigcup_{\theta_{k} \in\left(0, \theta^{*}\right]} \mathcal{F}_{\left(a, b, c, \theta_{k}\right)}^{-1}\left(D_{\theta_{k}}\right) \subseteq \bigcup_{\theta \in\left(0, \theta^{*}\right]} \mathcal{F}_{(a, b, c, \theta)}^{-1}\left(D_{\theta}\right) .
$$

By Condition 3.1, there exists a $\theta^{*} \in(0,1]$ such that the right-hand side of the above is bounded. However, the left-hand side is an unbounded sequence. This contradiction shows that the set (4.1) is indeed bounded. The proof is complete.

Since Condition 3.2 implies Condition 3.1, the following result is an immediate consequence of Theorem 4.1. 
COROllary 4.1. Let $f: R^{n} \rightarrow R^{n}$ be a continuous semimonotone function. If Condition 3.2 holds, then the set (4.1) is bounded.

While the system (2.3) has a solution for a continuous semimonotone function if Condition 3.1 holds, it is not clear if the solution of the system (2.3) is unique for each $\theta \in(0,1]$. However, for continuous $P_{0}$-functions which are special cases of continuous semimonotone functions, it is easy to prove that for each $\theta \in(0,1]$ the system (2.3) has a unique solution which is also continuous in $\theta$. We summarize the result as follows.

THEOREM 4.2. Let $f: R^{n} \rightarrow R^{n}$ be a continuous $P_{0}$-function.

(i) For each $\theta \in(0,1]$, the system $(2.3)$ has a unique solution $(u(\theta), x(\theta), y(\theta))$ which is continuous in $\theta$.

(ii) If Condition 3.1 (in particular, Condition 3.2) is satisfied, then the entire trajectory $\{(u(\theta), x(\theta), y(\theta)): \theta \in(0,1]\}$ is bounded. Hence, there exists at least a convergence subsequence $\left(u\left(\theta_{k}\right), x\left(\theta_{k}\right), y\left(\theta_{k}\right)\right)$ converging, as $\theta_{k} \rightarrow 0$, to $\left(0, x^{*}, y^{*}\right)$ where $x^{*}$ is a solution to the CP.

(iii) If $f$ is continuously differentiable, then $(u(\theta), x(\theta), y(\theta))$ is also continuously differentiable in $\theta$. In this case, the set $\{(u(\theta), x(\theta), y(\theta)): \theta \in(0,1]\}$ forms a smooth trajectory.

Proof. Since each $\mathrm{P}_{0}$-function is a semimonotone function, by Theorem 4.1 , the system (2.3) has at least one solution. It is sufficient to show that the system has at most one solution. Let

$$
g(x, a, c, \theta)=f(x)+\theta^{p} \operatorname{diag}\left(a^{p}\right) x+\theta c .
$$

Since $f$ is a $\mathrm{P}_{0}$-function and $a \in R_{++}^{n}$, the function $g(x, a, c, \theta)$ is a $\mathrm{P}$-function in $x$. Thus, by Lemma 3.3, the map

$$
\mathcal{Y}(x, \theta)=x+g(x, a, c, \theta)-\sqrt{(x-g(x, a, c, \theta))^{2}+4 \theta^{q} a^{q}}-\theta b
$$

is a $\mathrm{P}$-function in $x$. Since every P-function is univalent (one-to-one), the equation $\mathcal{Y}(x, \theta)=0$ has at most one solution. Hence, the system (2.3) has at most one solution by Lemma 2.1 .

The continuity of $(u(\theta), x(\theta), y(\theta))$ follows easily from Lemma 3.4. Indeed, given $\hat{\theta} \in(0,1)$, in order to show the continuity of $(u(\theta), x(\theta), y(\theta))$ at $\hat{\theta}$, it is sufficient to prove the continuity of $x(\theta)$ at $\hat{\theta}$. Since $\mathcal{Y}(x, \theta)$ is a P-function in $x, x(\hat{\theta})$ is the unique element in $\mathcal{Y}_{\hat{\theta}}^{-1}(0)=\{x: \mathcal{Y}(x, \hat{\theta})=0\}$. By Lemma 3.4, for any $\varepsilon>0$ there exists a scalar $\delta>0$ such that for any $\mathrm{P}_{0}$-function $h$ satisfying

$$
\sup _{x \in \bar{\Omega}}\|h(x)-\mathcal{Y}(x, \hat{\theta})\|<\delta,
$$

where $\Omega=\mathcal{Y}_{\hat{\theta}}^{-1}(0)+\varepsilon B$, then we have

$$
\emptyset \neq h^{-1}(0) \subseteq \mathcal{Y}_{\hat{\theta}}^{-1}(0)+\varepsilon B=x(\hat{\theta})+\varepsilon B .
$$

For this given $\delta$, it follows from (ii) of Lemma 3.2 that there is a scalar $\beta>0$ such that

$$
\sup _{x \in \bar{\Omega}}\|\mathcal{Y}(x, \theta)-\mathcal{Y}(x, \hat{\theta})\|<\delta
$$

for all $\theta>0$ such that $|\theta-\hat{\theta}|<\beta$. Setting $h(x):=\mathcal{Y}(x, \theta)$ in (4.4), we deduce from (4.5) that $\mathcal{Y}_{\theta}^{-1}(0)=\{x: \mathcal{Y}(x, \theta)=0\} \subseteq x(\hat{\theta})+\varepsilon B$ for all $\theta$ with $|\theta-\hat{\theta}|<\beta$. By 
the P-property of $\mathcal{Y}, x(\theta)$ is unique element in $\mathcal{Y}_{\theta}^{-1}(0)$. Thus, $\|x(\theta)-x(\hat{\theta})\|<\varepsilon$ for all $\theta>0$ such that $|\theta-\hat{\theta}|<\beta$, i.e., $x(\theta)$ is continuous at $\hat{\theta}$. Item (i) of the theorem follows.

Item (ii) follows immediately from Theorem 4.1 since $\mathrm{P}_{0}$-functions are semimonotone. We now prove Item (iii). Consider the following $3 n \times 3 n$ matrix

$$
A:=\left(\begin{array}{ccc}
I & 0 & 0 \\
-2 q U^{q-1} D & I-(X-Y) D & I+(X-Y) D \\
p U^{p-1} X & -\left(f^{\prime}(x)+\operatorname{diag}\left(u^{p}\right)\right) & I
\end{array}\right)
$$

where $U=\operatorname{diag}(u), X=\operatorname{diag}(x), Y=\operatorname{diag}(y)$ and $D=\operatorname{diag}(d)$ with $d=\left(d_{1}, \ldots, d_{n}\right)^{T}$ where

$$
d_{i}=1 / \sqrt{\left(x_{i}-y_{i}\right)^{2}+4 u_{i}^{q}}, i=1,2, \ldots, n .
$$

If $u \in R_{++}^{n}$, then it is easy to see that $I-(X-Y) D$ and $I+(X-Y) D$ are positive diagonal matrices for every $(x, y) \in R^{2 n}$. Thus, by Lemma 5.4 in Kojima et al. [19], the matrix

$$
\left(\begin{array}{cc}
I-(X-Y) D & I+(X-Y) D \\
-\left(f^{\prime}(x)+\operatorname{diag}\left(u^{p}\right)\right) & I
\end{array}\right)
$$

is nonsingular when $f$ is a $\mathrm{P}_{0}$-function. Hence $A$ is a nonsingular matrix for every $(u, x, y) \in R_{++}^{n} \times R^{2 n}$. Since the matrix $A$ coincides with the Jacobian matrix (with respect to $(u, x, y))$ of the equation

$$
H(u, x, y)-\theta(a, b, c)=0,
$$

by the implicit function theorem, there is a unique smooth (i.e., continuously differentiable) curve $(u(t), x(t), y(t))$ such that

$$
H(u(t), x(t), y(t))=t(a, b, c)
$$

for all $t$ sufficiently close to $\theta$ and

$$
\left.(u(t), x(t), y(t))\right|_{t=\theta}=(u(\theta), x(\theta), y(\theta)) .
$$

Particularly, $(u(\cdot), x(\cdot), y(\cdot))$ is continuously differentiable at $\theta$.

Furthermore, if $f$ is a $\mathrm{P}_{*}$-function, we can obtain a much stronger result. We now consider this important situation and show that for a $\mathrm{P}_{*}$-function the proposed trajectory exists and is bounded provided that the solution set of the CP is nonempty. For simplicity, we consider the case of $(a, b, c) \in R_{++}^{n} \times R_{-}^{n} \times R^{n}$, i.e., the vector $b$ is confined to $R_{-}^{n}$. We also consider the case of $c \in R_{++}^{n}$ when it is necessary. The stipulation that $b \in R_{-}^{n}$ has also been used in some non-interior-point algorithms, see Burke and $\mathrm{Xu}$ [3] and Hotta et al. [17], where the iterate $\left\{\left(x^{k}, y^{k}\right)\right\}$ is required to satisfy

$$
x^{k}+y^{k}-\sqrt{\left(x^{k}-y^{k}\right)^{2}+4 \mu^{k}} \leq 0
$$

which is equivalent to the requirement of " $b \in R_{-}^{n}$ ".

LEMmA 4.2. Let $v^{*}$ be an arbitrary solution of the $C P$ and $(a, b, c) \in R_{++}^{n} \times R_{-}^{n} \times$ $R^{n}$ be a fixed vector. Let $(u(\theta), x(\theta), y(\theta))$ satisfy the system (2.8) - (2.11) for each $\theta \in(0,1]$. Then, the following inequality holds.

$$
\left(x_{i}(\theta)-v_{i}^{*}\right)\left(f_{i}(x(\theta))-f_{i}\left(v^{*}\right)\right) \leq \theta^{q} e^{T} a^{q}-\theta b^{T} f\left(v^{*}\right) / 2-\theta^{p} \min _{1 \leq i \leq n} M_{i},
$$


where

$$
M_{i}=a_{i}^{p} x_{i}(\theta)\left(x_{i}(\theta)-v_{i}^{*}\right)+\theta^{1-p}\left(c_{i}-b_{i} / 2\right)\left(x_{i}(\theta)-v_{i}^{*}\right)
$$

Proof. Denote by $\bar{y}_{i}(\theta)=y_{i}(\theta)-\theta b_{i} / 2$ and $\bar{x}_{i}(\theta)=x_{i}(\theta)-\theta b_{i} / 2$. Let $v^{*}$ be an arbitrary solution to the CP. By $(2.11)$ and noting that $(\bar{x}(\theta), \bar{y}(\theta))>0$, we have for each $i$,

$$
\begin{aligned}
\left(\bar{y}_{i}(\theta)-f_{i}\left(v^{*}\right)\right)\left(\bar{x}_{i}(\theta)-v_{i}^{*}\right) & =\bar{y}_{i}(\theta) \bar{x}_{i}(\theta)-f_{i}\left(v^{*}\right) \bar{x}_{i}(\theta)-\bar{y}_{i}(\theta) v_{i}^{*} \\
& \leq \bar{y}_{i}(\theta) \bar{x}_{i}(\theta)=\theta^{q} a_{i}^{q} .
\end{aligned}
$$

We also note that

$$
\begin{aligned}
& \left(x_{i}(\theta)-v_{i}^{*}\right)\left(f_{i}(x(\theta))-f_{i}\left(v^{*}\right)\right) \\
= & \left(x_{i}(\theta)-v_{i}^{*}\right)\left(y_{i}(\theta)-\left(\theta a_{i}\right)^{p} x_{i}(\theta)-\theta c_{i}-f_{i}\left(v^{*}\right)\right) \\
= & \left(\bar{x}_{i}(\theta)-v_{i}^{*}+\theta b_{i} / 2\right)\left(\bar{y}_{i}(\theta)+\theta b_{i} / 2-\left(\theta a_{i}\right)^{p} x_{i}(\theta)-\theta c_{i}-f_{i}\left(v^{*}\right)\right) \\
= & \left(\bar{x}_{i}(\theta)-v_{i}^{*}\right)\left(\bar{y}_{i}(\theta)-f_{i}\left(v^{*}\right)\right)+\left(\bar{x}_{i}(\theta)-v_{i}^{*}\right)\left(\theta b_{i} / 2-\left(\theta a_{i}\right)^{p} x_{i}(\theta)-\theta c_{i}\right) \\
& +\left(\theta b_{i} / 2\right)\left(\bar{y}_{i}(\theta)+\theta b_{i} / 2-\left(\theta a_{i}\right)^{p} x_{i}(\theta)-\theta c_{i}-f_{i}\left(v^{*}\right)\right) .
\end{aligned}
$$

Thus, by (4.6) and noting that $b \leq 0$ and $\bar{y}(\theta)>0$, we have the following for all $i$

$$
\begin{aligned}
& \left(x_{i}(\theta)-v_{i}^{*}\right)\left(f_{i}\left(x(\theta)-f_{i}\left(v^{*}\right)\right)\right. \\
\leq & \theta^{q} a_{i}^{q}+\left(x_{i}(\theta)-v_{i}^{*}-\theta b_{i} / 2\right)\left(\theta b_{i} / 2-\left(\theta a_{i}\right)^{p} x_{i}(\theta)-\theta c_{i}\right) \\
& +\left(\theta b_{i} / 2\right)\left(\theta b_{i} / 2-\left(\theta a_{i}\right)^{p} x_{i}(\theta)-\theta c_{i}-f_{i}\left(v^{*}\right)\right) \\
= & \theta^{q} a_{i}^{q}-\left(\theta a_{i}\right)^{p} x_{i}(\theta)\left(x_{i}(\theta)-v_{i}^{*}\right) \\
& +\left(\theta b_{i} / 2-\theta c_{i}\right)\left(x_{i}(\theta)-v_{i}^{*}\right)-\theta b_{i} f_{i}\left(v^{*}\right) / 2 \\
\leq & \theta^{q} e^{T} a^{q}-\theta b^{T} f\left(v^{*}\right) / 2 \\
& -\theta^{p} \min _{1 \leq i \leq n}\left[a_{i}^{p} x_{i}(\theta)\left(x_{i}(\theta)-v_{i}^{*}\right)+\theta^{1-p}\left(c_{i}-b_{i} / 2\right)\left(x_{i}(\theta)-v_{i}^{*}\right)\right] .
\end{aligned}
$$

The last inequality follows from the fact that $a_{i}^{q} \leq e^{T} a^{q}$ and $-b_{i} f_{i}\left(v^{*}\right) \leq-b^{T} f\left(v^{*}\right)$ since $b \leq 0$ and $f\left(v^{*}\right) \geq 0$. The proof is complete.

We are ready for proving the following result.

THEOREM 4.3. Let $f$ be a continuous $P_{*}$-function and $(a, b, c) \in R_{++}^{n} \times R_{-}^{n} \times R^{n}$ be a fixed vector. Assume that the solution set of the CP is nonempty.

(i) If $p \leq 1$ and $q \in[1, \infty)$, then the trajectory $\{(u(\theta), x(\theta), y(\theta)): \theta \in(0,1]\}$ generated by (2.3) is bounded.

(ii) If $p>1, q \in[1, \infty)$ and $c \in R_{++}^{n}$, then the trajectory $\{(u(\theta), x(\theta), y(\theta)): \theta \in$ $(0,1]\}$ generated by $(2.3)$ is bounded.

Proof. We still use the notation

$$
(\bar{x}(\theta), \bar{y}(\theta))=(x(\theta)-\theta b / 2, y(\theta)-\theta b / 2) .
$$

By (2.11), and noting that $b \leq 0$ and $(\bar{x}(\theta), \bar{y}(\theta))>0$, we have

$$
\begin{aligned}
x(\theta)^{T} y(\theta) & =(\bar{x}(\theta)+\theta b / 2)^{T}(\bar{y}(\theta)+\theta b / 2) \\
& \leq \theta^{q} e^{T} a^{q}+\theta^{2}\|b\|^{2} / 4
\end{aligned}
$$


Let $v^{*}$ be an arbitrary solution of the CP. By the $\mathrm{P}_{*}$-property of $f$ and Lemma 4.2 , we have

$$
\begin{aligned}
& \left(v^{*}\right)^{T} y(\theta)+f\left(v^{*}\right)^{T} x(\theta) \\
= & -\left(x(\theta)-v^{*}\right)^{T}\left(y(\theta)-f\left(v^{*}\right)\right)+x(\theta)^{T} y(\theta) \\
= & -\left(x(\theta)-v^{*}\right)^{T}\left(f(x(\theta))+\theta^{p} \operatorname{diag}\left(a^{p}\right) x(\theta)+\theta c-f\left(v^{*}\right)\right)+x(\theta)^{T} y(\theta) \\
= & -\left(x(\theta)-v^{*}\right)^{T}\left(f(x(\theta))-f\left(v^{*}\right)\right)-\theta^{p}\left[\operatorname{diag}\left(a^{p}\right) x(\theta)\right]^{T}\left(x(\theta)-v^{*}\right) \\
& -\theta c^{T}\left(x(\theta)-v^{*}\right)+x(\theta)^{T} y(\theta) \\
\leq & \tau \sum_{i \in I_{+}}\left(x_{i}(\theta)-v_{i}^{*}\right)^{T}\left(f_{i}(x(\theta))-f_{i}\left(v^{*}\right)\right) \\
& -\theta^{p}\left[\operatorname{diag}\left(a^{p}\right) x(\theta)\right]^{T}\left(x(\theta)-v^{*}\right)-\theta c^{T}\left(x(\theta)-v^{*}\right)+x(\theta)^{T} y(\theta) \\
\leq & \tau n \max _{1 \leq i \leq n}\left(x_{i}(\theta)-v_{i}^{*}\right)^{T}\left(f_{i}(x(\theta))-f_{i}\left(v^{*}\right)\right) \\
& -\theta^{p}\left[\operatorname{diag}\left(a^{p}\right) x(\theta)\right]^{T}\left(x(\theta)-v^{*}\right)-\theta c^{T}\left(x(\theta)-v^{*}\right)+x(\theta)^{T} y(\theta) \\
\leq & \tau n\left(\theta^{q} e^{T} a^{q}-\theta b^{T} f\left(v^{*}\right) / 2-\theta^{p} \min _{1 \leq i \leq n} M_{i}\right) \\
& -\theta^{p}\left[\operatorname{diag}\left(a^{p}\right) x(\theta)\right]^{T}\left(x(\theta)-v^{*}\right)-\theta c^{T}\left(x(\theta)-v^{*}\right)+x(\theta)^{T} y(\theta) .
\end{aligned}
$$

The last inequality follows from Lemma 4.2 and $M_{i}$ is given as in Lemma 4.2. By (4.7) and the above inequality, we have

$$
\begin{aligned}
& \left(v^{*}\right)^{T} \bar{y}(\theta)+f\left(v^{*}\right)^{T} \bar{x}(\theta) \\
= & \left(v^{*}\right)^{T} y(\theta)+f\left(v^{*}\right)^{T} x(\theta)-\theta b^{T}\left(v^{*}+f\left(v^{*}\right)\right) / 2 \\
\leq & \theta^{q}(1+\tau n) e^{T} a^{q}+\theta^{2}\|b\|^{2} / 4-\theta \tau n b^{T} f\left(v^{*}\right) / 2-\theta^{p} \tau n \min _{1 \leq i \leq n} M_{i} \\
& -\theta^{p}\left[\operatorname{diag}\left(a^{p}\right) x(\theta)\right]^{T}\left(x(\theta)-v^{*}\right)-\theta c^{T}\left(x(\theta)-v^{*}\right)-\theta b^{T}\left(v^{*}+f\left(v^{*}\right)\right) / 2 .
\end{aligned}
$$

(i) We now consider the case of $p \leq 1$. Notice that the left-hand side is nonnegative. Dividing both sides of the above inequality by $\theta^{p}$ and rearranging terms we have

$$
\begin{aligned}
& {\left[\operatorname{diag}\left(a^{p}\right) x(\theta)\right]^{T}\left(x(\theta)-v^{*}\right)+\theta^{1-p} c^{T}\left(x(\theta)-v^{*}\right)+\tau n \min _{1 \leq i \leq n} M_{i}} \\
& \leq \theta^{q-p}(1+\tau n) e^{T} a^{q}+\theta^{2-p}\|b\|^{2} / 4-\theta^{1-p} \tau n b^{T} f\left(v^{*}\right) / 2 \\
& -\theta^{1-p} b^{T}\left(v^{*}+f\left(v^{*}\right)\right) / 2 .
\end{aligned}
$$

Since $a^{p} \in R_{++}^{n}, p \leq 1$ and $q \in[1, \infty)$, we conclude from the above inequality that the set $\{x(\theta): \theta \in(0,1]\}$ is bounded, and by continuity the set $\{y(\theta): \theta \in(0,1]\}$ is also bounded. Item (i) of the theorem is proved.

(ii) If $p>1$ and $c \in R_{++}^{n}$. In this case, since $(\bar{x}(\theta), \bar{y}(\theta))>0$ and $b \leq 0$, we have

$$
\begin{aligned}
M_{i} & \geq-a_{i}^{p} v_{i}^{*} x_{i}(\theta)+\theta^{1-p}\left(c_{i}-b_{i} / 2\right)\left(\bar{x}_{i}(\theta)+\theta b_{i} / 2-v_{i}^{*}\right) \\
& \geq-a_{i}^{p} v_{i}^{*} \bar{x}_{i}(\theta)+\theta^{1-p}\left(c_{i}-b_{i} / 2\right)\left(\theta b_{i} / 2-v_{i}^{*}\right) \\
& \geq-\left[\operatorname{diag}\left(a^{p}\right) \bar{x}(\theta)\right]^{T} v^{*}+\theta^{1-p} \min _{1 \leq i \leq n}\left(c_{i}-b_{i} / 2\right)\left(\theta b_{i} / 2-v_{i}^{*}\right) .
\end{aligned}
$$

Since the left-hand side of (4.8) is nonnegative, by (4.10) and (4.8), we have

$$
0 \leq \theta^{q}(1+\tau n) e^{T} a^{q}+\theta^{2}\|b\|^{2} / 4-\theta \tau n b^{T} f\left(v^{*}\right) / 2
$$




$$
\begin{aligned}
& +\theta^{p} \tau n\left[\operatorname{diag}\left(a^{p}\right) \bar{x}(\theta)\right]^{T} v^{*}-\theta \tau n \min _{1 \leq i \leq n}\left(c_{i}-b_{i} / 2\right)\left(\theta b_{i} / 2-v_{i}^{*}\right) \\
& +\theta^{p}\left[\operatorname{diag}\left(a^{p}\right) x(\theta)\right]^{T} v^{*}-\theta c^{T} x(\theta)+\theta c^{T} v^{*}-\theta b^{T}\left(v^{*}+f\left(v^{*}\right)\right) / 2 \\
\leq & \theta^{q}(1+\tau n) e^{T} a^{q}+\theta^{2}\|b\|^{2} / 4-\theta \tau n b^{T} f\left(v^{*}\right) / 2 \\
& +\theta^{p}(1+\tau n)\left[\operatorname{diag}\left(a^{p}\right) \bar{x}(\theta)\right]^{T} v^{*}-\theta \tau n \min _{1 \leq i \leq n}\left(c_{i}-b_{i} / 2\right)\left(\theta b_{i} / 2-v_{i}^{*}\right) \\
& +\theta^{p}\left[\operatorname{diag}\left(a^{p}\right) v^{*}\right]^{T} b / 2-\theta c^{T} \bar{x}(\theta)-\theta c^{T} b / 2+\theta c^{T} v^{*}-\theta b^{T}\left(v^{*}+f\left(v^{*}\right)\right) / 2 .
\end{aligned}
$$

Dividing both sides of the above by $\theta$ and rearranging terms, we have

$$
\begin{aligned}
& \left(c-\theta^{p-1}(1+\tau n) \operatorname{diag}\left(a^{p}\right) v^{*}\right)^{T} \bar{x}(\theta) \\
& \leq \theta^{q-1}(1+\tau n) e^{T} a^{q}+\theta\|b\|^{2} / 4-\tau n b^{T} f\left(v^{*}\right) / 2 \\
& -\tau n \min _{1 \leq i \leq n}\left(c_{i}-b_{i} / 2\right)\left(\theta b_{i} / 2-v_{i}^{*}\right) \\
& +\theta^{p-1}\left[\operatorname{diag}\left(a^{p}\right) v^{*}\right]^{T} b / 2-c^{T} b / 2+c^{T} v^{*}-b^{T}\left(v^{*}+f\left(v^{*}\right)\right) / 2
\end{aligned}
$$

Since $p>1$ and $c \in R_{++}^{n}$, there must exist a $\delta \in(0,1)$ such that for all $\theta \in(0, \delta]$, we have that $c-\theta^{p-1}(1+\tau n) \operatorname{diag}\left(a^{p}\right) v^{*} \geq c / 2>0$. Thus we can see from the above inequality that the set $\{\bar{x}(\theta): \theta \in(0, \delta]\}$ is bounded. Thus, the set $\{x(\theta): \theta \in(0, \delta]\}$ is bounded. The boundedness of the set $\{x(\theta): \theta \in[\delta, 1]\}$ can be obtained by (4.8) again. Indeed, if a subsequence in $\{x(\theta): \theta \in[\delta, 1]\}$ is unbounded, then there exists a subsequence denoted by $\left\{x\left(\theta_{k}\right)\right\}$ such that $\left\|x\left(\theta_{k}\right)\right\| \rightarrow \infty$ as $k \rightarrow \infty$ where $\theta_{k} \in[\delta, 1]$. Applying (4.8) to this sequence, the left-hand side of it is nonnegative. The right-hand side of the inequality (4.8), however, tends to $-\infty$. This is a contradiction. Therefore, we conclude that the entire set $\{x(\theta): \theta \in(0,1]\}$ is bounded. So is $\{y(\theta): \theta \in(0,1]\}$ by continuity.

Remark 4.1. The above result shows that the nonempty of the solution set implies the boundedness of the entire trajectory $\{(x(\theta), y(\theta)): \theta \in(0,1]\}$. Notice that the boundedness of this trajectory in turn implies the nonemptyness of the solution set. Therefore, we may conclude that the boundedness of this trajectory is equivalent to the solvability of the problem.

5. Limiting behavior of the trajectory. We have shown that Condition 3.1 (and hence most of the known conditions used in interior-point and non-interior-point methods) can guarantee the boundedness of the proposed continuation trajectory. Thus, there exists at least one convergent subsequence $\left\{\left(u\left(\theta_{k}\right), x\left(\theta_{k}\right), y\left(\theta_{k}\right)\right)\right\}$ whose limiting point is a solution to the CP. Two natural questions arise: (i) When is the entire trajectory convergent? (ii) What can be said about the limiting point of it? This section is devoted to these questions. For $0<p<1$ and $(a, b, c) \in R_{++}^{n} \times R_{-}^{n} \times R^{n}$ or $0<p \leq q$ and $(a, b, c) \in R_{++}^{n} \times\{0\} \times\{0\}$, we show (Theorems 5.1) that if $f$ is a $\mathrm{P}_{*}$-function and the $\mathrm{CP}$ has a least element solution, then the entire trajectory $\{(u(\theta), x(\theta), y(\theta))\}$ generated by $(2.3)$ converges, as $\theta \rightarrow 0$, to the unique least element solution, and that if $f$ is monotone, then the entire trajectory is convergent as $\theta \rightarrow 0$, and the limiting point is the $N$-norm least solution, where $N=\operatorname{diag}\left(a^{p}\right)$. For $p>q$ and $(a, b, c) \in R_{++}^{n} \times\{0\} \times\{0\}$, we show, among other things, that any limiting point of the sequence $\left\{\left(u\left(\theta_{k}\right), x\left(\theta_{k}\right), y\left(\theta_{k}\right)\right)\right\}$ as $\theta \rightarrow 0$ is a maximal complementarity solution (Theorem 5.2).

To begin, we recall some concepts that will be used in this section. An element $x^{*}$ of the set $S$ is said to be the $N$-norm least element, where $N$ is a positive definite matrix, if $\left\|N^{1 / 2} x^{*}\right\| \leq\left\|N^{1 / 2} u\right\|$ for all $u \in S$. Particularly, if $N=I$, the solution $x^{*}$ is 
called the least 2-norm element of $S$. An element $x^{*}$ of the set $S$ is said to be a least element of $S$ if $x^{*} \leq u$ for all $u \in S$ (Pang [24]). An element $x^{*}$ of the set $S$ is said to be a weak Pareto minimal element if there is no element $u$ in $S$ such that $u<x^{*}$ (Sznajder and Gowda [29]). It is evident that the (unique) least element is a weak Pareto minimal element, but the converse is not true. If the solution set $\mathrm{SOL}_{c p}(f)$ is convex, it is known that there exists a unique partition of the index set $\{1, \ldots, n\}$ denoted by $I, J$ and $O$ such that $\{1, \ldots, n\}=I \cup J \cup O$, and the intersection of each pair of them is empty. In fact,

$$
\begin{gathered}
I=\left\{i: x_{i}^{*}>0 \text { for some } x^{*} \in \mathrm{SOL}_{c p}(f)\right\} \\
J=\left\{j: f_{j}\left(x^{*}\right)>0 \text { for some } x^{*} \in \mathrm{SOL}_{c p}(f)\right\}, \\
O=\left\{k: x_{k}^{*}=f_{k}\left(x^{*}\right)=0, \text { for all } x^{*} \in \mathrm{SOL}_{c p}(f)\right\} .
\end{gathered}
$$

Since the solution set is convex, there must exist a solution $x^{*}$ satisfying $x_{i}^{*}>0$ for all $i \in I, f_{i}\left(x^{*}\right)>0$ for all $i \in J$ and $x_{i}^{*}=f_{i}\left(x^{*}\right)=0$ for all $i \in O$. Such a solution is called a maximal complementarity solution. When $O=\emptyset$, i.e., $x^{*}+f\left(x^{*}\right)>0, x^{*}$ is called a strict complementarity solution. We now prove the following result.

THEOREM 5.1. Assume that the solution set $S O L_{c p}(f)$ is nonempty. Let $p, q$ and $(a, b, c)$ satisfy one of the following conditions:

(C1) $0<p<1, q \in[1, \infty)$ and $(a, b, c) \in R_{++}^{n} \times R_{-}^{n} \times R^{n}$.

(C2) $0<p<q, q \in[1, \infty)$ and $(a, b, c) \in R_{++}^{n} \times\{0\} \times\{0\}$.

Then the following results hold:

(i) If $f$ is a continuous $P_{*}$-function and the least element solution of the $C P$ exists, then the entire trajectory $\{x(\theta): \theta \in(0,1]\}$ generated by (2.3) converges, as $\theta \rightarrow 0$, to the unique least element solution.

(ii) If $f$ is a continuous monotone mapping, then the entire continuation trajectory $\{x(\theta): \theta \in(0,1]\}$ generated by system (2.3) converges, as $\theta \rightarrow 0$, to a solution of the $C P$. This solution denoted by $x^{*}$ is a $N$-norm least solution, i.e.,

$$
\left\|N^{1 / 2} x^{*}\right\| \leq\left\|N^{1 / 2} v^{*}\right\| \text { for all } v^{*} \in S O L_{c p}(f),
$$

where $N=\operatorname{diag}\left(a^{p}\right)$. Particularly, if $a=\alpha e$ where $\alpha>0$ is a positive scalar, then this solution is the (unique) least 2-norm solution.

Proof. We show first the result holds under condition $(\mathrm{C} 1)$. Let $v^{*}$ be an arbitrary solution of the CP. Then, (4.9) holds. By (i) of Theorem 4.3 the entire continuation trajectory $\{(u(\theta), x(\theta), y(\theta))\}$ is bounded provided that the solution set of the CP is nonempty. Let $x^{*}$ be an arbitrary accumulation point of $\{x(\theta)\}$ as $\theta \rightarrow 0$. Since $0<p<1$ and $q \in[1, \infty)$, letting $\theta \rightarrow 0$ in (4.9), we have

$$
\tau n \min _{1 \leq i \leq n} a_{i}^{p} x_{i}^{*}\left(x_{i}^{*}-v_{i}^{*}\right)+\left[\operatorname{diag}\left(a^{p}\right)\left(x^{*}\right)\right]^{T}\left(x^{*}-v^{*}\right) \leq 0 .
$$

Note that $v^{*}$ is an arbitrary solution of the CP. If the problem has a least element solution $u^{*}$, setting $v^{*}=u^{*}$ in the above inequality, we deduce that $x^{*}=u^{*}$. Since the least element solution is unique, we conclude that the entire trajectory converges to the solution.

Since each monotone map is a $\mathrm{P}_{*}$-function with the constant $\tau=0$, the inequality (5.1), in this case, reduces to

$$
\left[\operatorname{diag}\left(a^{p}\right)\left(x^{*}\right)\right]^{T}\left(x^{*}-v^{*}\right) \leq 0,
$$


where $v^{*}$ is an arbitrary solution of the CP. To show the the convergence of the entire trajectory, it is sufficient to show that $x^{*}$ is unique. Indeed, if there exists another vector $u^{*}$ such that $u^{*}$ is also an accumulation point to the trajectory, then we have that

$$
\left[\operatorname{diag}\left(a^{p}\right)\left(u^{*}\right)\right]^{T}\left(u^{*}-v^{*}\right) \leq 0
$$

for all solution $v^{*}$. Since $x^{*}$ and $u^{*}$ are solutions to the CP, setting $v^{*}=u^{*}$ in $(5.2)$ and $v^{*}=x^{*}$ in (5.3), and adding the two inequalities, we obtain

$$
\left(x^{*}-u^{*}\right)^{T}\left[\operatorname{diag}\left(a^{p}\right)\right]\left(x^{*}-u^{*}\right) \leq 0 .
$$

Since $a^{p} \in R_{++}^{n}$, it follows from the above that $x^{*}=u^{*}$. Hence, the trajectory is convergent because it has a unique limiting point. It follows from (5.2) that

$$
\left\|N^{1 / 2} x^{*}\right\|^{2} \leq\left\|N^{1 / 2} x^{*}\right\|\left\|N^{1 / 2} v^{*}\right\|,
$$

where $N=\operatorname{diag}\left(u^{p}\right)$ and $v^{*}$ is an arbitrary solution of the CP. Therefore, $\left\|N^{1 / 2} x^{*}\right\| \leq$ $\left\|N^{1 / 2} v^{*}\right\|$ for any solution $v^{*}$, i.e., $x^{*}$ is a least $N$-norm solution. Particularly, if $a=\alpha e$ for some positive scalar $\alpha>0$, then (5.2) reduces to $\left(x^{*}\right)^{T}\left(x^{*}-v^{*}\right) \leq 0$ for all solution $v^{*}$, which implies that $x^{*}$ is the unique least 2-norm solution.

We now show the result under $(\mathrm{C} 2)$. It is evident that under condition $(\mathrm{C} 2)$, the inequality (4.8) can be written as:

$$
\begin{aligned}
\left(v^{*}\right)^{T} \bar{y}(\theta)+ & f\left(v^{*}\right)^{T} \bar{x}(\theta) \\
\leq & \theta^{q}(1+\tau n) e^{T} a^{q}-\theta^{p} \tau n \min _{1 \leq i \leq n} a_{i}^{p} x_{i}(\theta)\left(x_{i}(\theta)-x_{i}^{*}\right) \\
& -\theta^{p}\left[\operatorname{diag}\left(a^{p}\right) x(\theta)\right]^{T}\left(x(\theta)-v^{*}\right) .
\end{aligned}
$$

Dividing both sides of the above inequality by $\theta^{p}$ and noting that the left-hand side is nonnegative, we have

$$
\begin{aligned}
0 \leq & \theta^{q-p}(1+\tau n) e^{T} a^{q}-\tau n \min _{1 \leq n} a_{i}^{p} x_{i}(\theta)\left(x_{i}(\theta)-v_{i}^{*}\right) \\
& -\left[\operatorname{diag}\left(a^{p}\right) x(\theta)\right]^{T}\left(x(\theta)-v^{*}\right) .
\end{aligned}
$$

Since $p<q$, the above inequality implies that $\{x(\theta): \theta \in(0,1]\}$ is bounded, so is $\{y(\theta): \theta \in(0,1)\}$ by continuity. Let $x^{*}$ be an arbitrary accumulation point of $\{x(\theta)\}$ as $\theta \rightarrow 0$. Letting $\theta \rightarrow 0$ in the above inequality, we obtain the inequality (5.1) again. It suffices to repeat the proof of $(\mathrm{C} 1)$.

The above result states that when $p<q$ the trajectory of the monotone CP converges to a $N$-norm least solution. The next result studies the case of $p \geq q$.

THEOREM 5.2. Assume that $f$ is a monotone function.

(i) Let $p \geq q$ and $(a, b, c) \in R_{++}^{n} \times\{0\} \times\{0\}$. If the trajectory $\{x(\theta): \theta \in(0,1]\}$ generated by $(2.3)$ has an accumulation point as $\theta \rightarrow 0$, then any accumulation point of the trajectory, as $\theta \rightarrow 0$, is a maximal complementarity solution of the CP.

(ii) Let $p>q$ and $(a, b, c) \in R_{++}^{n} \times\{0\} \times\{0\}$. Assume that the $C P$ has a strict complementarity solution and the trajectory $\{x(\theta): \theta \in(0,1]\}$ generated by $(2.3)$ has an accumulation point as $\theta \rightarrow 0$. Then any accumulation point $(\hat{x}, \hat{y})$ of the trajectory as $\theta \rightarrow 0$ is a maximal strict complementarity solution in the sense that

$$
\sum_{i \in I} a_{i}^{q} \log v_{i}^{*}+\sum_{j \in J} a_{j}^{q} \log f_{j}\left(v^{*}\right) \leq \sum_{i \in I} a_{i}^{q} \log \hat{x}_{i}+\sum_{j \in J} a_{j}^{q} \log f_{j}(\hat{x}),
$$


where $v^{*}$ is an arbitrary strict complementarity solution. Furthermore, if $f$ is linear, i.e., $f=M x+u$ where $M$ is an $n$ by $n$ positive semi-definite matrix and $u \in R^{n}$ is a vector, then the entire trajectory converges, as $\theta \rightarrow 0$, to a unique maximal strict complementarity solution.

Proof. Since each accumulation point of the trajectory, as $\theta \rightarrow 0$, is a solution to the $\mathrm{CP}$, under the assumption of the theorem the solution set of the $\mathrm{CP}$ is nonempty. Let $v^{*}$ be an arbitrary solution to the CP. Thus, the inequality (4.8) remains valid. By assumption, we have $\tau=0$ and $b=c=0$. Therefore, (4.8) reduces to

$$
\begin{aligned}
\left(v^{*}\right)^{T} \bar{y}(\theta)+f\left(v^{*}\right)^{T} \bar{x}(\theta) & \leq \theta^{q} e^{T} a^{q}-\theta^{p}\left[\operatorname{diag}\left(a^{p}\right) x(\theta)\right]^{T}\left(x(\theta)-v^{*}\right) \\
& \leq \theta^{q} e^{T} a^{q}+\theta^{p}\left[\operatorname{diag}\left(a^{p}\right) x(\theta)\right]^{T} v^{*}
\end{aligned}
$$

Notice that the solution set of a monotone $\mathrm{CP}$ is convex. Let $I, J, O$ be the unique partition of the indexes $\{1,2, \ldots, n\}$ as defined at the beginning of this section. Then the above inequality further reduces to

$$
\left(v^{*}\right)_{I}^{T} \bar{y}_{I}(\theta)+f_{J}\left(v^{*}\right)^{T} \bar{x}_{J}(\theta) \leq \theta^{q} e^{T} a^{q}+\theta^{p}\left[\operatorname{diag}\left(a_{I}^{p}\right) x_{I}(\theta)\right]^{T} v_{I}^{*} .
$$

Since $(\bar{x}(\theta), \bar{y}(\theta)) \in R_{++}^{2 n}$, we have

$$
\begin{aligned}
& \left(v^{*}\right)_{I}^{T} \bar{X}_{I}^{-1}(\theta) \bar{X}_{I}(\theta) \bar{y}_{I}(\theta)+f_{J}\left(v^{*}\right)^{T} \bar{Y}_{J}^{-1}(\theta) \bar{Y}_{J}(\theta) \bar{x}_{J}(\theta) \\
& \leq \theta^{q} e^{T} a^{q}+\theta^{p}\left[\operatorname{diag}\left(a_{I}^{p}\right) x_{I}(\theta)\right]^{T} v_{I}^{*}
\end{aligned}
$$

where $\bar{X}_{I}(\theta)=\operatorname{diag}\left(\bar{x}_{I}(\theta)\right)$ and $\bar{Y}_{J}(\theta)=\operatorname{diag}\left(\bar{y}_{J}(\theta)\right)$. Since

$$
\bar{X}_{I}(\theta) \bar{y}_{I}(\theta)=\theta^{q} a_{I}^{q}, \bar{X}_{J}(\theta) \bar{y}_{J}(\theta)=\theta^{q} a_{J}^{q},
$$

from the above inequality we have

$$
\left(v^{*}\right)_{I}^{T} \bar{X}_{I}^{-1}(\theta) a_{I}^{q}+f_{J}\left(v^{*}\right)^{T} \bar{Y}_{J}^{-1}(\theta) a_{J}^{q} \leq e^{T} a^{q}+\theta^{p-q}\left[\operatorname{diag}\left(a_{I}^{p}\right) x_{I}(\theta)\right]^{T} v_{I}^{*} .
$$

The above inequality holds for all solution $v^{*}$. Particularly, let $v^{*}$ be a solution satisfying $v_{I}^{*}>0$ and $f_{J}\left(v^{*}\right)>0$, i.e., let $v^{*}$ be a maximal complementarity solution. Assume that $(\hat{x}, \hat{y})$ is an accumulation point of $(x(\theta), y(\theta))$ as $\theta \rightarrow 0$. Taking $\theta \rightarrow 0$ in the above inequality we deduce that $\hat{x}_{I}>0$ and $\hat{y}_{J}>0$ since $p \geq q$. Notice that $(\hat{x}, \hat{y})$ is a solution of the CP. $(\hat{x}, \hat{y})$ is a maximal complementarity solution. Result (i) follows.

We now prove the result (ii). Let $v^{*}$ in (5.4) be an arbitrary strict complementarity solution. Assume that $(\hat{x}, \hat{y})$ is an arbitrary accumulation point of $\{x(\theta), y(\theta))$ as $\theta \rightarrow 0$. We now prove that $(\hat{x}, \hat{y})$ is a strict complementarity solution. Taking the limit in (5.4) and noting that $p>q$, we deduce that $\hat{x}_{I}>0, \hat{y}_{J}>0$ and that

$$
\left(v^{*}\right)_{I}^{T} \hat{X}_{I}^{-1}(\theta) a_{I}^{q}+f_{J}\left(v^{*}\right)^{T} a t Y_{I}^{-1}(\theta) a_{J}^{q} \leq e^{T} a^{q} .
$$

Notice that $\hat{y}=f(\hat{x})$. The above inequality can be further written as

$$
\sum_{i \in I} a_{i}^{q}\left(v_{i}^{*} / \hat{x}_{i}\right)+\sum_{j \in J} a_{j}^{q}\left(f_{j}\left(v^{*}\right) / f_{j}(\hat{y})\right) \leq e^{T} a^{q} .
$$

Since $1+\log t \leq t$ for all $t>0$, from the above we have

$$
\sum_{i \in I} a_{i}^{q}\left[1+\log \left(v_{i}^{*} / \hat{x}_{i}\right)\right]+\sum_{j \in J} a_{j}^{q}\left[1+\log \left(f_{j}\left(v^{*}\right) / f_{j}(\hat{y})\right)\right] \leq e^{T} a^{q} .
$$


Since a strict complementarity solution exists, we have $I \cup J=\{1, \ldots, n\}$, and thus

$$
\sum_{i \in I} a_{i}^{q} \log \left(v_{i}^{*} / \hat{x}_{i}\right)+\sum_{j \in J} a_{j}^{q} \log \left(f_{j}\left(v^{*}\right) / f_{j}(\hat{y})\right) \leq 0 .
$$

Therefore,

$$
\sum_{i \in I} a_{i}^{q} \log v_{i}^{*}+\sum_{j \in J} a_{j}^{q} \log f_{j}\left(v^{*}\right) \leq \sum_{i \in I} a_{i}^{q} \log \hat{x}_{i}+\sum_{j \in J} a_{j}^{q} \log f_{j}(\hat{y}) .
$$

Since $v^{*}$ is an arbitrary strict complementarity solution, the first part of result (ii) is proved.

We now consider the linear case, i.e., $f=M x+u$. Denote by SSOL $(f)$ the set of strict complementarity solutions of the $\mathrm{CP}$, which is also a convex set by the convexity of $\mathrm{SOL}_{c p}(f)$. Since $f$ is linear, this fact in turn implies that the following set is also convex:

$$
\bar{S}=\{(x, y): y=M x+u, x \in \operatorname{SSOL}(f)\} .
$$

To show the second part of result (ii), it is sufficient to prove that the accumulation point $(\hat{x}, \hat{y})$ satisfying $(5.5)$ is unique. In fact, it is easy to see that $(\hat{x}, \hat{y})$ is the solution to the following strict concave program:

$$
\begin{aligned}
& \text { Maximize } \sum_{i \in I} a_{i}^{q} \log x_{i}+\sum_{i \in J} a_{j}^{q} \log y_{i} \\
& \text { subject to }(x, y) \in \bar{S}
\end{aligned}
$$

Since a strict concave program has at most one solution, $(\hat{x}, \hat{y})$ is the unique solution to the above program, which is a maximal strict complementarity solution of the CP. Thus the entire trajectory is convergent.

We close this section by proving a general result concerning the characterization of the limiting point of the trajectory proposed in this paper in the case of semimonotone functions.

THEOREM 5.3. Let $f$ be a continuous semimonotone function from $R^{n}$ into $R^{n}$. Let $p, q$ and $(a, b, c)$ satisfy one of the following conditions:

(C1) $0<p<1, q \in[1, \infty)$ and $(a, b, c) \in R_{++}^{n} \times R^{2 n}$.

(C2) $0<p<q, q \in[1, \infty)$ and $(a, b, c) \in R_{++}^{n} \times\{0\} \times\{0\}$.

Let $(u(\theta), x(\theta), y(\theta))$ be a solution to the system $(2.3)$ for each $\theta \in(0,1]$. Assume that there exists an accumulation point to the trajectory $(u(\theta), x(\theta), y(\theta))$ as $\theta \rightarrow 0$. Then for any accumulation point $\left(0, x^{*}, y^{*}\right)$ of this trajectory as $\theta \rightarrow 0, x^{*}$ is a weak Pareto minimal solution to the CP.

Proof. let $\left(0, x^{*}, y^{*}\right)$ be an arbitrary accumulation point of $(u(\theta), x(\theta), y(\theta))$ as $\theta \rightarrow 0$. Then there exists a subsequence $\left\{\theta_{k}\right\} \rightarrow 0$ such that

$$
\left\{\left(u\left(\theta_{k}\right), x\left(\theta_{k}\right), y\left(\theta_{k}\right)\right)\right\} \rightarrow\left(0, x^{*}, y^{*}\right) .
$$

Assume the contrary that $x^{*}$ is not a weak Pareto minimal solution. Then there exists a solution $u^{*}$ satisfying $u^{*}<x^{*}$. Since $x\left(\theta_{k}\right) \rightarrow x^{*}$, we have $x\left(\theta_{k}\right)>u^{*}$ for all sufficiently large $k$. By the semimonotone property of $f$, for each sufficiently large $k$ there is an index $i_{k}$ such that

$$
x_{i_{k}}\left(\theta_{k}\right)>u_{i_{k}}^{*} \text { and } f_{i_{k}}\left(x\left(\theta_{k}\right)\right) \geq f_{i_{k}}\left(u^{*}\right) .
$$


Passing through a subsequence, we may assume that there exists an index $l$ such that

$$
x_{l}\left(\theta_{k}\right)>u_{l}^{*} \text { and } f_{l}\left(x\left(\theta_{k}\right)\right) \geq f_{l}\left(u^{*}\right)
$$

for all sufficiently large $k$. Notice that for each $\theta$, the solution $(u(\theta), x(\theta), y(\theta))$ of the system (9) satisfies the system (2.8)-(2.11). We still use the symbols of $\bar{y}_{i}(\theta)=$ $y_{i}(\theta)-\theta b_{i} / 2>0$ and $\bar{x}_{i}(\theta)=x_{i}(\theta)-\theta b_{i} / 2>0$. By (4.6) we have

$$
\left(\bar{y}_{l}\left(\theta_{k}\right)-f_{l}\left(u^{*}\right)\right)\left(\bar{x}_{l}\left(\theta_{k}\right)-u_{l}^{*}\right) \leq \theta_{k}^{q} a_{l}^{q} .
$$

On the other hand, we have

$$
\begin{aligned}
& \left(\bar{y}_{l}\left(\theta_{k}\right)-f_{l}\left(u^{*}\right)\right)\left(\bar{x}_{l}\left(\theta_{k}\right)-u_{l}^{*}\right) \\
= & \left(\bar{y}_{l}\left(\theta_{k}\right)-f_{l}\left(u^{*}\right)\right)\left(-\theta_{k} b_{l} / 2\right)+\left(\bar{y}_{l}\left(\theta_{k}\right)-f_{l}\left(u^{*}\right)\right)\left(x_{l}\left(\theta_{k}\right)-u_{l}^{*}\right) \\
= & \left(\bar{y}_{l}\left(\theta_{k}\right)-f_{l}\left(u^{*}\right)\right)\left(-\theta_{k} b_{l} / 2\right) \\
& +\left(f_{l}\left(x\left(\theta_{k}\right)\right)+\theta_{k}^{p} a_{l}^{p} x_{l}\left(\theta_{k}\right)+\theta_{k} c_{l}-\theta_{k} b_{l} / 2-f_{l}\left(u^{*}\right)\right)\left(x_{l}\left(\theta_{k}\right)-u_{l}^{*}\right) \\
= & \left(y_{l}\left(\theta_{k}\right)-\theta_{k} b_{l} / 2-f_{l}\left(u^{*}\right)\right)\left(-\theta_{k} b_{l} / 2\right) \\
& +\left(f_{l}\left(x\left(\theta_{k}\right)\right)-f_{l}\left(u^{*}\right)\right)\left(x_{l}\left(\theta_{k}\right)-u_{l}^{*}\right) \\
& +\left(\theta_{k}^{p} a_{l}^{p} x_{l}\left(\theta_{k}\right)+\theta_{k} c_{l}-\theta_{k} b_{l} / 2\right)\left(x_{l}\left(\theta_{k}\right)-u_{l}^{*}\right) \\
\geq & \left(y_{l}\left(\theta_{k}\right)-\theta_{k} b_{l} / 2-f_{l}\left(u^{*}\right)\right)\left(-\theta_{k} b_{l} / 2\right) \\
& +\left(\theta_{k}^{p} a_{l}^{p} x_{l}\left(\theta_{k}\right)+\theta_{k} c_{l}-\theta_{k} b_{l} / 2\right)\left(x_{l}\left(\theta_{k}\right)-u_{l}^{*}\right) .
\end{aligned}
$$

Combining the above two inequalities and dividing both sides by $\theta_{k}^{p}$, we have

$$
\begin{aligned}
\theta_{k}^{q-p} a_{l}^{2} \geq & \left(y_{l}\left(\theta_{k}\right)-\theta_{k} b_{l} / 2-f_{l}\left(u^{*}\right)\right)\left(-\theta_{k}^{1-p} b_{l} / 2\right) \\
& +\left(a_{l}^{p} x_{l}\left(\theta_{k}\right)+\theta_{k}^{1-p} c_{l}-\theta_{k}^{1-p} b_{l} / 2\right)\left(x_{l}\left(\theta_{k}\right)-u_{l}^{*}\right) .
\end{aligned}
$$

Let $\theta_{k} \rightarrow 0$. It is easy to see that under either condition (C1) or (C2) we have

$$
a_{l}^{p} x_{l}^{*}\left(x_{l}^{*}-u_{l}^{*}\right) \leq 0
$$

which contradicts the assumption $0 \leq u^{*}<x^{*}$.

6. Final remarks. We have proved the existence and the boundedness of a new homotopy continuation trajectory for nonlinear $\mathrm{P}_{0}$-complementarity problems. The assumption imposed in the paper is weaker than most existing conditions widely used in interior-point and non-interior-point methods. Particularly, this assumption is satisfied if the $\mathrm{P}_{0}$-CP has a nonempty and bounded solution set. Therefore, the method proposed in this paper can tackle all $\mathrm{P}_{0}-\mathrm{CPs}$ with bounded solution sets. Since this assumption can be satisfied even when the strict feasibility condition fails to hold, the proposed method can also be used to tackle some $\mathrm{P}_{0}-\mathrm{CPs}$ with unbounded solution sets. For $\mathrm{P}_{*}$-CPs, the existence and the boundedness of the new continuation trajectory can be guaranteed provided that the solution set of the CP is nonempty (no matter whether the solution set is bounded or not). Moreover, under some choices of $p, q$ and $(a, b, c)$, the entire trajectory for any continuous monotone $\mathrm{CP}$ always converges to a solution of the $\mathrm{CP}$ provided that a solution exists. Based on the results of this paper, we may design a non-interior-point path-following algorithm for CPs, which can solve all solvable $\mathrm{P}_{*}$-CPs, all $\mathrm{P}_{0}$-CPs with bounded solution sets, and some $\mathrm{P}_{0}$-CPs with unbounded solution sets. 
Since the proposed method can deal with all solvable $\mathrm{P}_{*}$-CPs, a natural question is whether this method can attack all solvable $\mathrm{P}_{0}$-CPs. That is, can the Condition 3.1 in Theorem 4.2 be replaced by the nonemptyness assumption of the solution set? The answer is no. For some $\mathrm{P}_{0}-\mathrm{CPs}$ with unbounded solution sets, the proposed continuation trajectory might be divergent to infinity. This phenomena has also been seen in the canonical Tikhonov regularization trajectory for a $\mathrm{P}_{0}-\mathrm{CP}$ with an unbounded solution set (Sznajder and Gowda [29]). In fact, in section 3.4 of [20], Kojima et al. pointed out that certain knapsack problem can be transformed into a linear $\mathrm{P}_{0}-\mathrm{CP}$ with an unbounded solution set. For this $\mathrm{P}_{0}-\mathrm{CP}$, it is easy to very that both the canonical Tikhonov regularization trajectory and the continuation trajectory proposed in this paper is divergent to infinity. This implies that the example in section 3.4 of [20] does not satisfy Condition 3.1.

\section{REFERENCES}

[1] J. V. Burke and S. Xu, The global linear convergence of a non-interior path-following algorithm for linear complementarity problems, Math. Oper. Res., 23 (1998), pp. 719-734.

[2] J. V. Burke and S. Xu, A non-interior predictor-corrector path-following method for LCP, Reformulation: Nonsmooth, Piecewise Smooth, Semismooth and Smoothing Methods, Appl. Optim., 22, Kluwer Acad. Publ., Dordrecht, 1999, pp. 25-63.

[3] J. V. Burke and S. Xu, A non-interior predictor-corrector path following algorithm for the monotone linear complementarity problem, Math. Programming, 87 (2000), pp. 113130.

[4] J. V. Burke and S. Xu, The Complexity of A Non-Interior-Path Following Methods for the Linear Complementarity Problem, Report, Department of Mathematics, University of Washington, Seattle, 1999.

[5] B. Chen and P. T. Harker, A non-interior-point continuation method for linear complementarity problems, SIAM J. Matrix Anal. Appl., 14 (1993), pp. 1168-1190.

[6] B. Chen and X. Chen, A global and local superlinear continuation method for $P_{0}$ and $R_{0}$ and monotone NCP, SIAM J. Optim., 9 (1999), pp. 624-645.

[7] B. Chen, X. Chen and C. Kanzow, A Penalized Fischer-Burmeister NCP-Function: Theoretical Investigation and Numerical Results, Math. Programming, 88 (2000), pp. 211-216.

[8] R. W. Cottle, J. S. Pang and R. E. Stone, The Linear Complementarity Problem, Academic Press, Boston, 1992.

[9] R. W. Cottle, J. S. Pang and V. Venkateswaran, Sufficient matrices and the linear complementarity problems, Linear Algebra Appl., 114/115 (1989), pp. 231-249.

[10] F. Facchinei, Structural and stability properties of $P_{0}$ nonlinear complementarity problems, Math. Oper. Res., 23 (1998), pp. 735-745.

[11] F. Facchinei and C. Kanzow, Beyond monotonicity in regularization methods for nonlinear complementarity problems, SIAM J. Control Optim., 37 (1999), pp. 1150-1161.

[12] F. Facchinei and J.S. Pang, Total Stability of Variational Inequalities, Dipartimento di Informatica e Sistemistica, Università di Roma, Via Buonarroti, Roma, Italy, 1998.

[13] M. S. Gowda and M. A. Tawhid, Existence and limiting behavior of trajectories associated with $P_{0}$-equations, Comput. Optim. Appl., 12 (1999), pp. 229-251.

[14] P. T. Harker and J. S. Pang, Finite-dimensional variational inequality and nonlinear complementarity problems: a survey of theory, algorithms and applications, Math. Programming, Ser. B, 48 (1990), pp. 161-220.

[15] W. P. M. H. Heemels, J. M. Schumacher and S. Weiland, Linear complementarity systems, SIAM J. Appl. Math. 60 (2000), pp. 1234-1269.

[16] K. Hotta and A. Yoshise, Global convergence of a class of non-interior-point algorithms using Chen-Harker-Kanzow-functions for nonlinear complementarity problems, Math. Programming, 86 (1999), pp. 105-133.

[17] K. Hotta, M. Inaba and A. Yoshise, A complexity analysis of a smoothing method using CHKS-function for monotone linear complementarity problems, Comput. Optim. Appl., 17 (2000), 183-201.

[18] C. Kanzow, Some nonlinear continuation methods for linear complementarity problems, SIAM J. Matrix Anal. Appl., 17 (1996), pp. 851-865.

[19] M. Kojima, N. Megiddo, and T. Noma, Homotopy continuation methods for nonlinear com- 
plementarity problems, Math. Oper. Res., 16 (1991), pp. 754-774.

[20] M. Kojima, N. Megiddo, T. Noma and A. Yoshise, A unified approach to interior point algorithms for linear complementarity problems, Lecture Notes in Computer Sciences, 538, Springer-Verlag, New York, 1991.

[21] M. Kojima, M. Mizuno, and T. Noma, Limiting behavior of trajectories generated by a continuation method for monotone complementarity problems, Math. Oper. Res., 43 (1990), pp. 662-675.

[22] L. Lloyd, Degree Theory, Cambridge University Press, Cambridge, England, 1978.

[23] P. Lötstedt, Mechanical systems of rigid bodies subject to unilateral constraints, SIAM J. Appl. Math., 42 (1982), pp. 281-296.

[24] J. S. Pang, Least Element Complementarity Theory, Ph.D. Thesis, Department of Operations Research, Stanford University, Stanford, California, 1976.

[25] H. D. Qi, Tikhonov regularization methods for variational inequality problems, J. Optim. Theory Appl., 102 (1999), pp. 193-201.

[26] L. Qi and D. Sun, Improving the convergence of non-interior point algorithm for nonlinear complementarity problems, Math. Comp., 69 (2000), pp. 283-304.

[27] G. Ravindran and M. S. Gowda, Regularization of $P_{0}$-functions in box variational inequality problems, SIAM J. Optim., 11 (2000), pp. 748-760.

[28] D. Sun, A regularization Newton method for solving nonlinear complementarity problems, Appl. Math. Optim., 40 (1999), pp. 315-339.

[29] R. Sznajder and M. S. Gowda, On the limiting behavior of the trajectory of regularized solutions of $P_{0}$ complementarity problems, Reformulation: Nonsmooth, Piecewise Smooth, Semismooth and Smoothing Methods, Appl. Optim., 22, Kluwer Academic Publishers, pp. 371-379, 1999.

[30] P. Tseng, Growth behavior of a class of merit functions for the nonlinear complementarity problems, J. Optim. Theory Appl., 89 (1996), pp. 17-37.

[31] P. Tseng, Error bounds for regularized complementarity problems, Ill-Posed Variational Problems and Regularization Techniques (Trier, 1998), Lecture Notes in Econom. and Math., Systems, 477, Springer, Berlin, pp. 247-274.

[32] P. Tseng, Analysis of a non-interior continuation method based on Chen-Mangasarian smoothing functions for complementarity problems, Reformulation: Nonsmooth, Piecewise Smooth, Semismooth and Smoothing Methods, Appl. Optim., 22, Kluwer Acad. Publ., Dordrecht, pp. 381-404, 1999.

[33] H. Väliaho, $P_{*}$ matrices are just sufficient, Linear Algebra Appl., 239 (1996), pp. 103-108.

[34] V. Venkateswaran, An algorithm for the linear complementarity problem with a $P_{0}$-matrix, SIAM J. Matrix Anal. Appl., 14 (1993), pp. 967-977.

[35] S. Xu, The Global Linear Convergence and Complexity of a Non-Interior Path-Following Algorithm for Monotone LCP Based on Chen-Harker-Kanzow-Smale Smoothing Function, Report, Department of Mathematics, University of Washington, Seattle, 1997.

[36] S. Xu and J. V. Burke, A polynomial time interior-point path-following algorithm for LCP based on Chen-Harker-Kanzow smoothing techniques, Math. Programming, 86 (1999), pp. 91-103.

[37] A. J. van der Schaft and J. M. Schumacher, Complementarity modeling of hybrid systems, IEEE Tran. Auto. Control, 43 (1998), pp. 483-490.

[38] Y. B. Zhao and J. Han, Two interior-point methods for nonlinear $P_{*}(\tau)$-complementarity problems, J. Optim. Theory Appl., 102 (1999), pp. 659-679.

[39] Y. B. Zhao and G. Isac, Quasi- $P_{*}$-maps, $P(\tau, \alpha, \beta)$-maps, exceptional family of elements and complementarity problems, J. Optim. Theory Appl., 105 (2000), pp. 213-231.

[40] Y. B. Zhao and G. Isac, Properties of a multi-valued mapping associated with some nonmonotone complementarity problems, SIAM J. Control Optim., 39 (2000), pp. 571-593.

[41] Y. B. Zhao and D. Li, Strict feasibility conditions in complementarity problems, J. Optim. Theory Appl., 107 (2000), pp. 641-664.

[42] Y. B. Zhao and D. Li, On a new homotopy continuation trajectory for nonlinear complementarity problems, Math. Oper. Res., 26 (2001), pp. 119-146. 